\title{
Psicooncología
}

ISSN: 1696-7240

\section{Propiedades Psicométricas del Cuestionario de Motivos a Favor y en Contra de la Reconstrucción Mamaria}

\author{
José Moral de la Rubia ${ }^{1, *}$; Melina Miaja Avila ${ }^{2}$; Paulina Bajonero Canónico ${ }^{3}$; Cynthia \\ Villarreal Garza ${ }^{4}$
}

Recibido: 30 de marzo de 2019 / aceptado: 5 de septiembre de 2019

Resumen: Antecedentes: Aunque la reconstrucción mamaria (RM) tras la mastectomía es una opción estética con buenos resultados, se realiza poco en México. Para estudiar sus razones se creó el Cuestionario de Motivos a favor y en contra de la Reconstrucción Mamaria (CMRM). La de validez de contenido del CMRM ha sido comprobada, pero no otras propiedades psicométricas. Objetivos: determinar su estructura factorial y comprobar su fiabilidad y validez de constructo concurrente. Método: Este estudio instrumental con diseño no experimental de corte transversal usó un muestreo intencional. Se aplicó el CMRM integrado por dos escalas, un cuestionario de imagen corporal y preguntas sobre datos sociodemográficos, clínicos y conducta sexual a 110 mujeres mexicanas mastectomizadas. Resultados: La escala de motivos a favor de la RM del CMRM presentó una estructura unidimensional, consistencia interna excelente y validez convergente. La escala de motivos en contra de la RM presentó una estructura de tres factores: miedos/preocupaciones/costos, aceptación/satisfacción con el estado actual y falta de información. Sus tres factores presentaron consistencia interna y validez convergente y discriminante. La correlación entre ambas escalas fue pequeña, pero la consistencia interna del CMRM fue excelente. La fuerza de asociación del CMRM fue muy fuerte con deseo de RM, media con edad e imagen corporal y pequeña con deseo de conservar la mama, frecuencias de relaciones sexuales antes del diagnóstico, búsqueda de información sobre RM y percepción del estado económico. Fue independiente de escolaridad, etapa del cáncer, tipo de mastectomía y estado civil. Conclusión: el cuestionario es fiable y válido.

Palabras clave: Cáncer de mama, mastectomía, reconstrucción mamaria, motivos, imagen corporal.

\section{[en] Psychometric Properties of the Questionnaire of Motives for and against Breast Reconstruction}

\footnotetext{
Abstract: Backgrounds: Although breast reconstruction (BR) after mastectomy is an aesthetic option with good results, it is done little in Mexico. To study its reasons, the Questionnaire of Motives for and against Breast Reconstruction (QMBR) was created. The content validity of the QMBR has been tested,

1 José Moral de la Rubia. Facultad de Psicología, Universidad Autónoma de Nuevo León. Nuevo León. México. E-mail: jose_moral@hotmail.com

2 Melina Miaja Ávila. Tecnologico de Monterrey, Centro de Cáncer de Mama. Nuevo León. México. E-mail:miajaam@live.com.mx

3 Paulina Bajonero Canónico. Tecnologico de Monterrey, Escuela de Medicina y Ciencias de la Salud. E-mail: pau.bajoneer@gmail.com

4 Cynthia Villarreal Garza. Tecnologico de Monterrey, Centro de Cáncer de Mama. Nuevo León. México E-mail: cynthiavg@gmail.com

* Dirección de correspondencia: José Moral de la Rubia. Dr. Carlos Canseco 110. Col. Mitras Centro. Monterrey, NL, México. E-mail: jose_moral@hotmail.com
} 
but not other psychometric properties. The objectives of the study were to determine its factor structure, and to test its reliability and concurrent construct validity. This instrumental research with nonexperimental cross-sectional design used an intentional sampling. The QMBR consisting of two scales, a body image questionnaire, and questions about sociodemographic, clinical and sexual behavior data were applied to 110 Mexican mastectomized women. The QMBR scale of motives for BR presented a one-factor structure, excellent internal consistency, and convergent validity. The QMBR scale of motives against BR presented a structure of three factors: fears/concerns/cost, acceptance/satisfaction with the current state, and lack of information. Its three factors showed internal consistency and convergent validity and discriminant. The correlation between both scales was small, but the internal consistency of QMBR was excellent. The strength of association of QMBR was very strong with desire for BR; medium with age and body image; and small with desire to preserve the breast, frequencies of sexual relations before diagnosis, search for information on BR, and perception of economic status. The QMBR was independent of levels of education, stage of cancer, type of mastectomy, and marital status. It is concluded that the questionnaire is reliable and valid.

Keywords: Breast cancer, mastectomy, breast reconstruction, motives, body image.

Sumario. 1. Introducción 2. Método 3. Análisis estadístico 4. Resultados 4.1.Estructura factorial, consistencia interna y validez convergente y discriminante 4.2. Relación con variables clínicas, imagen corporal, frecuencia de relaciones sexuales y variables sociodemográficas 5. Discusión 6. Referencias bibliográficas

Cómo citar: Moral de la Rubia J, Miaja Ávila M, Bajonero Canónico P, Villarreal Garza C. Propiedades Psicométricas del Cuestionario de Motivos a Favor y en Contra de la Reconstrucción Mamaria. Psicooncología 2016; 287-313. doi:10.5209/psic.65592

\section{Introducción}

Mohar et al.(1) informaron que el $81 \%$ de las mujeres diagnosticadas con cáncer de mama en el Instituto Nacional de Cancerología en México requirieron una mastectomía. En las mujeres intervenidas con mastectomía, la RM juega un papel muy importante. En este sentido, se ha reportado que la RM puede mejorar en gran medida diferentes aspectos de la vida de las mujeres, como son una mayor satisfacción con la imagen corporal, vida sexual, bienestar psicosocial y funcionalidad física en comparación con las mujeres que no se sometieron a la cirugía reconstructiva $a^{(2,3)}$. Asimismo, la RM puede disminuir en un $36 \%$ el riesgo de desarrollar linfedema ${ }^{(4)}$.

En la actualidad existe una amplia gama de opciones de RM; no obstante, las tasas de estas cirugías siguen siendo muy bajas en todos los países. La tasa es del $12 \%$ en Australia ${ }^{(5)}, 16,5 \%$ en Inglaterra ${ }^{(6)}, 18,6 \%$ en Colombia ${ }^{(7)}, 21-29,2 \%$ en Estados Unidos $^{(8,9)}, 21 \%$ en Suecia ${ }^{(10)}$ y $32,1 \%$ en Francia ${ }^{(11)}$. En México, no se cuenta con suficientes datos, pero se estima que el porcentaje es ínfimo. En este sentido, López, Romeo, Bracqbien y Ramos ${ }^{(12)}$ informaron que de 101 mujeres mastectomizadas entre el 2010 y 2012, únicamente tres de ellas se habían realizado la RM. Por ende, cabría preguntarnos: ¿a qué se debe que las tasas de RM sean tan bajas?, y ¿qué motiva a las pacientes para optar o no por realizarse la RM?

Los motivos por los cuales las mujeres con mastectomía se realizan o no la RM han sido estudiados desde finales de los noventas ${ }^{(13)}$, y estas investigaciones han ido en aumento con mayor ímpetu en países de la Unión Europea ${ }^{(11,14-17)}$. Los resultados son similares a lo largo del tiempo. Dichos estudios han desarrollado instrumentos para medir los motivos por los cuales las mujeres con mastectomía se someten o 
no a una RM. Sin embargo, estos se han creado exclusivamente para cumplir con los objetivos de su estudio, sin pretender validar los mismos ${ }^{(11,14-18)}$. Por ejemplo, Hequet et al. ${ }^{(11)}$ y Zieliński et al. ${ }^{(17)}$ desarrollaron cuestionarios de opción múltiple para conocer los motivos por los cuales las mujeres con mastectomía decidieron no realizarse la RM. En ambos estudios, no queda claro cómo se crearon las preguntas que conforman los cuestionarios, ni la cantidad de preguntas que los conforman. Además, en ninguno de ellos se validó el instrumento de medida creado.

Es importante contar con instrumentos fiables y validados en población mexicana que permitan homologar sus hallazgos con los de estudios similares y aportar evidencias científicas a los profesionales de la salud que requieran conocer los motivos a favor y en contra de la RM. Estos datos favorecerán una mejor atención clínica, al tomar decisiones conjuntas y congruentes con las necesidades específicas de las mujeres mastectomizadas. A su vez, posibilitarían el desarrollo de modelos acerca de los factores que subyacen a estas motivaciones.

Para tal fin, Miaja, Moral y Villarreal( ${ }^{(19)}$ desarrollaron el CMRM integrado por dos escalas. El enfoque de su desarrollo fue empírico. Se basó en la exploración de los argumentos expresados por las pacientes. Estos se obtuvieron a través de una técnica de redes semánticas naturales. Desde estos datos se elaboraron 27 ítems y a continuación su comprensibilidad y adecuación fue valorada por un juicio de expertos. Se realizaron modificaciones en la redacción de ítems y se añadieron dos más con base en el juicio de expertos. El cuestionario finalmente quedó integrado por 16 ítems para la escala de motivos a favor de la RM (EMAF) y 13 ítems para la escala de motivos en contra de la RM (EMEC). El CMRM mide en qué grado las mujeres mastectomizadas están a favor o en contra de realizarse la RM, es decir, evalúa su disposición motivacional hacia la $\mathrm{RM}^{(19)}$.

Los objetivos de esta investigación son: 1) describir la distribución y comprobar la discriminabilidad y fiabilidad por consistencia interna de los ítems de la CMRM, 2) determinar su estructura factorial, verificando la consistencia interna del cuestionario, escalas y factores (grado de precisión al medir el constructo con distintos indicadores), la validez convergente de cada modelo de medida (grado de certeza en los indicadores miden un mismo constructo) y validez discriminante entre los factores (grado de certeza en que las covarianzas entre los ítems de un factor son mayores que las covarianzas con los ítems de los otros factores), 3) describir la distribución de las puntuaciones, y 4) estudiar su relación con variables clínicas, imagen corporal, frecuencia de relaciones sexuales y variables sociodemográficas para aportar evidencias de validez de constructo concurrente.

\section{Método}

Se realizó un estudio instrumental. Su diseño fue no experimental de corte transversal.

\section{Participantes}

Los criterios de inclusión fueron: ser mujer con diagnóstico de cáncer de mama, haber sido intervenida con mastectomía, ser mayor de edad y estar bajo tratamiento oncológico. Los criterios de exclusión fueron: no conceder el consentimiento informado para la aplicación del cuestionario, tener déficits intelectuales o cognitivos 
que impidiesen responder al cuestionario de auto-informe. Los criterios de eliminación fueron: cuestionario incompleto o datos de expediente médico incompleto.

Se contactó con 116 mujeres con diagnóstico de cáncer de mama, mastectomizadas y atendidas en el Centro de Cáncer de Mama del Hospital San José. En todos los casos, los gastos médicos eran cubiertos por el Seguro Popular. Una de ellas rehusó dar el consentimiento informado, tres casos fueron eliminados por datos incompletos en el cuestionario y dos por datos incompletos en su expediente médico. La muestra analizada fue de 110 mujeres, el $91 \%$ con mastectomía unilateral y $9 \%$ bilateral. Al preguntar si deseaba conservar la mama, $66,4 \%$ dijeron que sí y $33,6 \%$ no, estos mismos porcentajes se encontraron al preguntar si desea o no hacerse la RM. La relación entre estas dos variables fue significativa $\left(\chi^{2}[1, N=110]=9,080, p=0,003\right.$, $\varphi=0,308)$. El deseo de conservar la mama aumenta 3,9 veces la probabilidad de quererse hacer la $\mathrm{RM}$ en comparación que no desear conservar la mama: $\mathrm{OR}=3,88$, IC $95 \%(1,67 ; 9,01)$.

La media de edad fue 50,80, IC 95\% $(48,73 ; 52,87)$, con una desviación estándar de 10,96 , variando de 30 a 78 . La escolaridad varió de $0=$ ninguna a $8=$ profesional terminada. Su mediana correspondió a $4=$ secundaria terminada $\left(Q_{1}=1=\right.$ primaria truncada y $\mathrm{Q}_{3}=6=$ media superior terminada). Con respecto a la ocupación, el 67,3\% indicaron ser amas de casa, $18,2 \%$ tenían contratos de tiempo completo, 9,1\% de medio tiempo, $4,5 \%$ estaban desempleadas y $0,9 \%$ incapacitadas. El ingreso familiar mensual promedio varió de $1=$ menos de 3.000 pesos a $6=$ más de 48.000 pesos. Su mediana correspondió a $2=3.000$ a $5.999\left(\mathrm{Q}_{1}=1=\right.$ " $<3.000$ " y $\mathrm{Q}_{3}=3=$ "6.000 a 11.999"). Al preguntar la percepción de su estado económico actual, el 50\% reportó tener dificultades para cubrir sus gastos, $33,6 \%$ tener poco dinero para gastos extras, $11,8 \%$ tener dinero para cubrir sus gastos familiares y $4,5 \%$ tener dinero suficiente para gastos extras. En el momento de la entrevista, 48,2\% de las mujeres estaban casadas, $16,4 \%$ en unión libre, $12,7 \%$ separadas o divorciadas, $11,8 \%$ solteras y $10,9 \%$ viudas.

\section{Instrumento de medida}

Se aplicó el CMRM de Miaja et al. ${ }^{(19)}$, el Cuestionario de Imagen Corporal (CIC) ${ }^{(20)}$ y preguntas sobre información sociodemográfica, clínica y frecuencia de relaciones sexuales.

El CMRM está integrado por dos escalas: la EMAF con 16 ítems redactados a favor de la RM y la EMEC con 13 ítems redactados en contra de la RM. Los ítems se responden en una escala tipo Likert con siete categorías ordenadas de respuesta que varían de 1 = "totalmente en desacuerdo" a 7 = "totalmente de acuerdo". La puntuación en cada escala se obtiene a través del cociente entre la suma de sus ítems y el número de ítems sumados, por lo que el rango varía de 1 a 7 . Se calcula una puntuación para el cuestionario a través de diferencia entre ambas escalas (EMAF EMEC) y su rango es de -7 a 7. Una puntuación más positiva en CMRM refleja una disposición motivacional más favorable hacia la $\mathrm{RM}^{(19)}$.

ElCIC consta de 12 ítems, que se responden en escala tipo Likert con cuatro opciones que va de $1=$ "nada" a 4 = "mucho". Mayor puntuación refleja mayor problemática relacionada con la imagen corporal. En una muestra de 188 mujeres españolas con cáncer de mama, su estructura factorial fue de un factor y su fiabilidad a través de la consistencia interna fue buena $(\alpha=0,88)^{(20)}$. En la presente muestra, la consistencia interna de los 12 ítems fue buena ( $\alpha$ de Cronbach $=0,81$ y $\alpha$ ordinal $=0,86$ ). 


\section{Procedimiento}

De forma previa a la consulta se identificaron a las pacientes con diagnóstico de cáncer de mama que habían sido intervenidas con una mastectomía entre todas las pacientes atendidas en el Centro de Cáncer de Mama del hospital San José. Durante la consulta se invitó a estas pacientes a participar en el estudio. Las pacientes que desearon participar firmaron el consentimiento informado. Tras la consulta respondían por escrito al cuestionario de autorreporte en la sala de infusión o en sala de espera.

El protocolo fue aprobado por los comités de ética y de investigación de la escuela de medicina del Tecnológico de Monterrey y de la Fundación Santos y de la Garza Evia (con número de registro ante la comisión nacional de bioética CONBIOETICA19CEI00820130520), apegándose a las normas éticas de investigación en humanos ${ }^{(21-23)}$.

\section{Análisis de datos}

Los análisis se hicieron por escala. El cuestionario se consideró como un compuesto de ambas escalas. Se comprobó la discriminabilidad de los ítems de la EMAF y EMEC por la correlación entre el ítem y su escala dicotomizada en puntuaciones bajas ( $\leq$ percentil 27 ) y altas ( $\geq$ percentil 73 ), calculada a través del coeficiente de correlación biserial por rangos. La fiabilidad del ítem se estimó por dos índices: el índice de homogeneidad o correlación del ítem con la suma de los ítems restantes de su escala y el efecto de la eliminación del ítem sobre la consistencia interna de su escala. La correlación se calculó a través del coeficiente de correlación poliserial. La consistencia interna de la escala se calculó por el coeficiente alfa ordinal. Se retuvieron aquellos ítems sin efecto suelo o techo (porcentaje de primera o última categoría menor que $80 \%$ ), con valores de correlación (para la discriminabilidad y el índice de homogeneidad) mayores que 0,35 y que provocasen un descenso en el valor del coeficiente alfa ordinal de su escala al ser excluidos ${ }^{(24)}$.

Para determinar la estructura factorial de cada escala se usó análisis factorial exploratorio (AFE) y confirmatorio (AFC) aplicado sobre la misma muestra. Este último se hizo como un análisis complementario para estimar los índices de ajuste ${ }^{(25)}$. Para ambos análisis se usó la matriz de correlación policórica.

En el AFE, el número de ítems se determinó por la convergencia entre cinco criterios empíricos: análisis paralelo (con 2.000 muestras aleatorias generadas por permutación de datos, punto de corte: percentil 95), coordenadas óptimas (dependiente del análisis paralelo previo), media mínima de las correlaciones parciales al cuadrado y a la cuarta potencia y el número de autovalores mayores que uno ${ }^{(26)}$. Los factores se extrajeron por mínimos cuadrados ponderados. La matriz de cargas factoriales se rotó por el método Promax. Se retuvieron los ítems con cargas factoriales mayores que 0,40 en un factor ${ }^{(25)}$.

En el AFC, la función de discrepancia se optimizó por mínimos cuadrados simples o libres de escala. El ajuste se valoró por medio de ocho índices: chicuadrada relativa $\left(\chi^{2} / \mathrm{gl}\right)$, índice de bondad de ajuste (GFI) y su variante ajustada (AGFI), índice normado de ajuste (NFI), índice relativo de ajuste (RFI), índice comparativo de ajuste (CFI), error de aproximación cuadrático medio (RMSEA) y residuo estandarizado cuadrático medio (SRMR). Siguiendo a Byrne ${ }^{(27)}$, se consideró 
que el ajuste es bueno cuando $\chi^{2} / \mathrm{gl} \leq 2$, GFI, NFI, RFI y CFI $\geq 0,95, \mathrm{AGFI} \geq 0,90, \mathrm{y}$ RMSEA y SRMR $\leq 0,05$; aceptable $\chi^{2} / \mathrm{gl} \leq 3$, GFI, NFI, RFI y CFI $\geq 0,90$, AGFI $\geq$ 0,85 , y RMSEA y SRMR $<0,10$.

La validez convergente de cada factor se estimó por la varianza media extraída (AVE) y el coeficiente omega de McDonald. En el AFE, ambos se calcularon desde la matriz de cargas estructurales. Valores de AVE $>0,50$ y $\omega \geq 0,70$ indican validez convergente. La validez discriminante entre cada par de factores se comprobó por una varianza compartida entre los dos factores menor que el AVE de cada factor y menor que dos tercios ${ }^{(28)}$.

La consistencia interna de los factores y las dos escalas se estimaron por el coeficiente alfa ordinal ${ }^{(29)}$. Al estar integrado el cuestionario por dos escalas, su consistencia interna se calculó por el coeficiente alfa estratificado ${ }^{(30)}$. Valores de $\alpha$ ordinal y $\alpha_{\text {estratificado }}<0,50$ se consideraron que muestran una consistencia interna inaceptable, entre 0,50 a 0,59 muy baja, entre 0,60 a 0,69 cuestionable, entre 0,70 a 0,79 aceptable, entre 0,80 a 0,89 buena $y \geq 0,90$ excelente $^{(29,30)}$,

El ajuste de las puntuaciones a una distribución normal se contrastó por las pruebas de Kolmogorov-Smirnov con la correlación de Lilliefors y de D'AgostinoPearson. En caso de mantenerse la hipótesis nula por ambas pruebas con una $\mathrm{p}>$ 0,05 , se habla de normalidad y aproximación a la normalidad cuando se mantiene con una $\mathrm{p}>0,05$ por la prueba de D'Agostino-Pearson y $\mathrm{p}>0,01$ por la prueba de Kolmogorov-Smirnov-Lilliefors.

La relación con variables clínicas, imagen corporal, frecuencia de relaciones sexuales y variables sociodemográficas se calcularon por el coeficiente de correlación producto-momento de Pearson, correlación poliserial, correlación biserial por rangos, correlación por rangos de Spearman y coeficiente eta dependiendo de la escala de medida y la normalidad distribucional. Correlaciones entre 0,10 y 0,30 se consideró que reflejan una fuerza de asociación pequeña, entre 0,30 y0,49 media, entre 0,50 y 0,69 grande, entre 0,70 y 0,89 muy grande $y \geq 0,90$ unitaria $^{(31)}$.

\section{Resultados}

Distribución, discriminabilidad y fiabilidad de los items de la EMAF y EMEC

En la EMAF, ningún ítem mostró efecto suelo o techo. Las frecuencias más altas aparecieron en los dos valores extremos y las más bajas en los valores centrales, mostrando un perfil en forma de U. Todos los ítems presentaron asimetría negativa o cola larga hacia los valores bajos $\left(\mathrm{A}_{\mathrm{IQ}}<-0,10\right)$, salvo el ítem 13 que fue simétrico. Todos ellos fueron discriminativos $\left(\mathrm{r}_{\mathrm{br}} \geq 0,79\right)$ y presentaron fiabilidad $\left(\mathrm{r}_{\mathrm{PSi}, \mathrm{t}-\mathrm{i}}, \mathrm{O} \mathrm{r}_{\text {Si,t-i }}\right.$ $\geq 0,67$ y $\mathrm{O} \alpha_{\mathrm{t}-\mathrm{i}}<\mathrm{O} \alpha_{\mathrm{t}}$ ), por lo que cumplieron con los criterios de retención (Tabla 1).

En la EMEC, ningún ítem presentó efecto suelo o techo. Al igual que en la EMAF, el perfil de la distribución de los ítems tomó una forma de U. Todos los ítems fueron discriminativos $\left(\mathrm{r}_{\mathrm{br}} \geq 0,45\right)$ y cumplieron con el primer criterio de fiabilidad $\left(\mathrm{r}_{\mathrm{PSi}, \mathrm{t}-\mathrm{i}}>0,35\right)$. Con la excepción del ítem $18\left(\mathrm{O} \alpha_{\mathrm{t}-\mathrm{i}}=0,894\right)$, también cumplieron con el segundo criterio de fiabilidad $\left(\mathrm{O} \alpha_{\mathrm{t}-\mathrm{i}}<\mathrm{O} \alpha_{\mathrm{t}}^{\mathrm{t}}=0,891\right)$ (Tabla 1). No obstante, se decidió retener el ítem 18 por la importancia de su contenido (preocupaciones económicas) y cumplir el criterio de discriminabilidad y uno de los criterios de fiabilidad. 
Tabla 1. Porcentajes de las categorías de respuesta, distribución, discriminabilidad y fiabilidad de los ítems de la EMAF y EMEC

\begin{tabular}{|c|c|c|c|c|c|c|c|c|c|c|c|c|c|c|c|}
\hline \multirow{2}{*}{ It } & \multicolumn{7}{|c|}{ Porcentajes (\%) } & \multicolumn{5}{|c|}{ Descriptivos } & \multirow{2}{*}{$\frac{\text { Dis }}{r_{b r}}$} & \multicolumn{2}{|c|}{ Fiabilidad } \\
\hline & TD & $\mathrm{BD}$ & $\mathrm{AD}$ & $\mathrm{N}$ & AA & BA & TA & Mo & $\mathrm{Me}$ & $\mathrm{R}_{\mathrm{SI}}$ & $\mathrm{A}_{\mathrm{IQ}}$ & $\mathrm{C}_{\mathrm{PC}}$ & & $\mathrm{r}_{\mathrm{PS}}$ & $\mathrm{O} \alpha_{\mathrm{t}-\mathrm{i}}$ \\
\hline \multicolumn{16}{|c|}{ Motivos a favor de la $\mathrm{RM}\left(\mathrm{O} \alpha_{\mathrm{t}}=0,980\right)$} \\
\hline 1 & 32,7 & 7,3 & 8,2 & 10,9 & 15,5 & 10,9 & 14,5 & 1 & 4 & 2,5 &,- 20 &,- 05 &, 85 & ,77 & ,979 \\
\hline 2 & 28,2 & 4,5 & 0,9 & 9,1 & 20,9 & 10 & 26,4 & 1 & 5 & 3 &,- 33 &,- 01 & ,91 &, 84 & ,978 \\
\hline 3 & 42,7 & 1,8 & 6,4 & 9,1 & 10,9 & 7,3 & 21,8 & 1 & 3 & 2,5 & ,20 &,- 05 & ,86 & ,78 & 979 \\
\hline 4 & 32,7 & 3,6 & 0 & 9,1 & 15,5 & 10 & 29,1 & 1 & 5 & 3 &,- 33 &,- 01 & ,94 & ,88 & ,978 \\
\hline 5 & 31,8 & 2,7 & 4,5 & 8,2 & 16,4 & 15,5 & 20,9 & 1 & 5 & 2,5 &,- 60 &,- 05 & ,87 & ,74 & ,980 \\
\hline 6 & 37,3 & 6,4 & 4,5 & 12,7 & 10,9 & 10 & 18,2 & 1 & 4 & 2,5 &,- 20 &,- 05 & ,79 & 67 & ,981 \\
\hline 7 & 32,7 & 4,5 & 4,5 & 7,3 & 11,8 & 13,6 & 25,5 & 1 & 5 & 3 &,- 33 &,- 01 & 94 & ,84 & 978 \\
\hline 8 & 25,5 & 1,8 & 1,8 & 4,5 & 13,6 & 8,2 & 44,5 & 7 & 6 & 3 &,- 67 &,- 01 & ,94 & ,81 & ,978 \\
\hline 9 & 27,3 & 3,6 & 0 & 7,3 & 14,5 & 10,9 & 36,4 & 7 & 5 & 3 &,- 33 &,- 01 & 94 & ,87 & 978 \\
\hline 10 & 30,9 & 1,8 & 1,8 & 9,1 & 10 & 10,9 & 35,5 & 7 & 5 & 3 &,- 33 &,- 01 & ,96 &, $87^{\mathrm{s}}$ & ,977, \\
\hline 11 & 26,4 & 1,8 & 0 & 8,2 & 15,5 & 10 & 38,2 & 7 & 5 & 3 &,- 33 &,- 01 & ,94 & ,83 & ,978 \\
\hline 12 & 36,4 & 1,8 & 1,8 & 2,7 & 11,8 & 12,7 & 32,7 & 1 & 5 & 3 &,- 33 &,- 01 & 97 &, $90^{\mathrm{s}}$ & 977 \\
\hline 13 & 34,5 & 5,5 & 2,7 & 10,9 & 11,8 & 6,4 & 28,2 & 1 & 4 & 3 & ,00 &,- 01 & ,93 & ,86 & ,978 \\
\hline 14 & 40 & 5,5 & 2,7 & 12,7 & 10 & 5,5 & 23,6 & 1 & 4 & 2,5 &,- 20 &,- 05 & ,88 & ,77 & 979 \\
\hline 15 & 30 & 3,6 & 0,9 & 8,2 & 15,5 & 10 & 31,8 & 7 & 5 & 3 &,- 33 &,- 01 & ,81 & ,75 & ,979 \\
\hline 16 & 42,7 & 4,5 & 0,9 & 18,2 & 6,4 & 4,5 & 22,7 & 1 & 4 & 2,5 &,- 20 &,- 05 &, 84 & ,81 & 979, \\
\hline \multicolumn{16}{|c|}{ Motivos contra la $\mathrm{RM}\left(\mathrm{O} \alpha_{\mathrm{t}}=0,891\right)$} \\
\hline 17 & 26,4 & 6,4 & 6,4 & 2,7 & 10 & 10 & 38,2 & 7 & 5 & 3 &,- 33 &,- 01 & ,69 &, 56 & ,882 \\
\hline 18 & 17,3 & 2,7 & 4,5 & 3,6 & 5,5 & 18,2 & 48,2 & 7 & 6 & 1,625 &,- 38 &,- 13 & ,46 & ,37 & 894 \\
\hline 19 & 24,5 & 9,1 & 7,3 & 10 & 15,5 & 5,5 & 28,2 & 7 & 4 & 2,625 &, 14 &,- 04 &, 72 &, 57 & ,882 \\
\hline 20 & 22,7 & 5,5 & 5,5 & 12,7 & 10 & 12,7 & 30,9 & 7 & 5 & 2,5 &,- 20 &,- 05 &, 56 & ,48 & 884 \\
\hline 21 & 23,6 & 5,5 & 9,1 & 13,6 & 6,4 & 11,8 & 30 & 7 & 4 & 2,5 & ,20 &,- 05 & ,45 & ,38 & 889 \\
\hline 22 & 26,4 & 10 & 6,4 & 12,7 & 6,4 & 12,7 & 25,5 & 1 & 4 & 3 &, 00 &,- 01 & ,77 & ,62 & ,878 \\
\hline 23 & 30 & 4,5 & 5,5 & 11,8 & 10,9 & 9,1 & 28,2 & 1 & 4 & 3 &, 00 &,- 01 & ,76 & ,62 & 880 \\
\hline 24 & 40 & 6,4 & 7,3 & 13,6 & 5,5 & 7,3 & 20 & 1 & 3 & 2,5 & ,20 &,- 05 & ,61 &, 51 & 886 \\
\hline 25 & 27,3 & 2,7 & 5,5 & 10,9 & 8,2 & 17,3 & 28,2 & 7 & 5 & 3 &,- 33 &,- 01 & ,90 &, 75 & 873 \\
\hline 26 & 26,4 & 3,6 & 6,4 & 4,5 & 9,1 & 11,8 & 38,2 & 7 & 5,5 & 3 &,- 50 &,- 01 &, 84 & ,65 & 877 \\
\hline 27 & 50 & 2,7 & 4,5 & 11,8 & 3,6 & 9,1 & 18,2 & 1 & 1,5 & 2,5 & 80 &,- 05 & ,71 & ,61 & ,882 \\
\hline 28 & 22,7 & 4,5 & 3,6 & 8,2 & 13,6 & 14,5 & 32,7 & 7 & 5 & 2,5 &,- 20 &,- 05 & ,59 & ,49 & 887 \\
\hline 29 & 20 & 3,6 & 2,7 & 6,4 & 12,7 & 11,8 & 42,7 & 7 & 6 & 2 &,- 50 &,- 10 &, 52 &, 50 & 886 \\
\hline
\end{tabular}

Notas: Categorías ordenadas de respuestas: TD "totalmente en desacuerdo" = 1, BD "bastante en desacuerdo" $=2, \mathrm{AD}$ "algo en desacuerdo" = 3, N "ni de acuerdo ni en desacuerdo" = 4, AA "algo de acuerdo" = 5, BA "bastante de acuerdo" = 6 y TA "totalmente de acuerdo" $=7$. Descriptivos: $\mathrm{Mo}=$ moda, $\mathrm{Me}=$ mediana, $\mathrm{R}_{\mathrm{SI}}=$ rango semiintercuartílico, $\mathrm{A}_{\mathrm{IQ}}=$ coeficiente de asimetría intercuartílico de Bowley, $\mathrm{y}_{\mathrm{PC}}=$ coeficiente de curtosis percentílico corregido. Discriminabilidad: $\mathrm{r}_{\mathrm{br}}=$ correlación biserial por rangos entre el ítem y la escala dicotomizada en grupo de puntuaciones bajas ( $\leq$ percentil 27 ) y altas ( $\geq$ percentil 73 ). Fiabilidad: $r_{P S}=$ coeficiente de correlación poliserial del ítem con la escala excluido el ítem y $\mathrm{O} \alpha_{\mathrm{t}-\mathrm{i}}=$ coeficiente alfa ordinal de la escala excluido el ítem. 


\subsection{Estructura factorial, consistencia interna $y$ validez convergente $y$ discriminante}

\section{$E M A F$}

El número de factores fue uno por el análisis paralelo, coordenadas óptimas, media mínima de las correlaciones parciales a la cuarta potencia y número de autovalores mayores que 1 en la matriz de correlación policórica. La media mínima de las correlaciones parciales al cuadrado indicó dos factores.

Al extraer un factor por mínimos cuadrados ponderados, las cargas variaron de 0,71 a 0,97 con una media de 0,87 , Su consistencia interna fue excelente ( $\alpha$ ordinal $=0,98)$. La varianza media extraída fue alta $(\mathrm{AVE}=0,77)$, al igual que la fiabilidad compuesta $(\omega=0,98)$, por lo que el factor presentó validez convergente.

Al especificar un modelo de un factor de motivos a favor de la RM con 16 indicadores (ítems 1-16), la solución convergió en 12 iteraciones y fue admisible. Todos los parámetros al ser estimado por intervalo con un nivel de confianza al 95\% por el método de percentiles corregidos de sesgo fueron significativos. El factor mostró validez convergente. El ajuste del modelo fue bueno (Figura 1 y Tabla 2).

Figura 1. Modelo de un factor de motivos a favor de la RM estimado por SLS

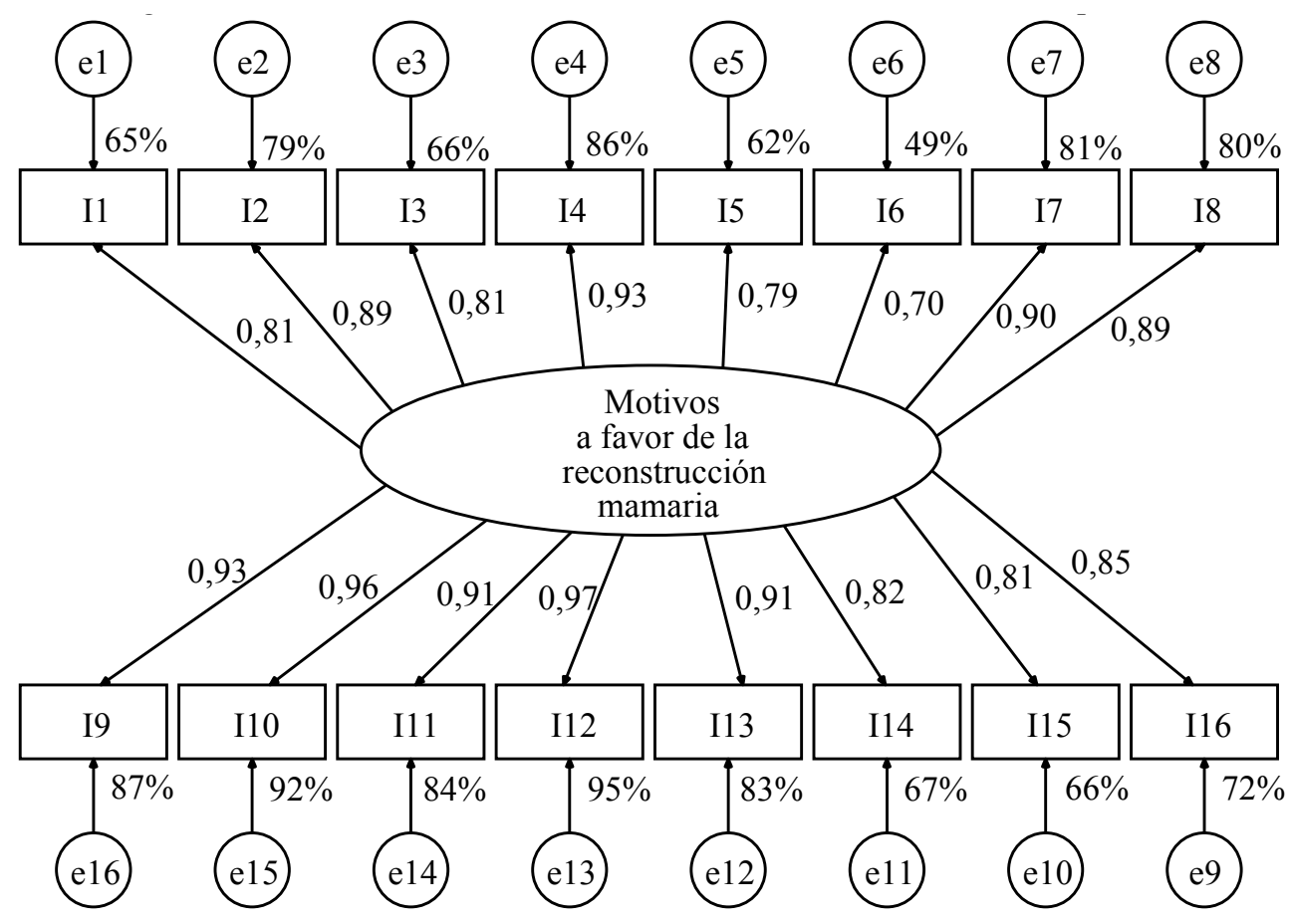


Tabla 2. Índices de ajuste de los modelos factoriales para EMAF, EMEC y el cuestionario y validez convergente de sus factores

\begin{tabular}{|c|c|c|c|c|c|}
\hline & \multirow{2}{*}{$\begin{array}{l}\text { Índices } \\
\text { de ajuste }\end{array}$} & EMAF & \multicolumn{2}{|c|}{ EMEC } & \multirow{2}{*}{$\begin{array}{c}\text { Cuestionario } \\
4 \mathrm{~F}-29 \mathrm{i}\end{array}$} \\
\hline & & 1F_16i & $3 \mathrm{~F}_{-} 13 \mathrm{i}$ & $2 F_{-} 13 \mathrm{i}$ & \\
\hline \multicolumn{2}{|r|}{$\chi^{2}$} & 38,494 & 81,166 & 123,243 & 470,642 \\
\hline \multicolumn{2}{|r|}{$\mathrm{gl}$} & 104 & 62 & 64 & 373 \\
\hline \multicolumn{2}{|r|}{$\chi^{2 / g l}$} & 0,370 & 1,309 & 1,926 & 1,262 \\
\hline \multicolumn{2}{|r|}{ GFI } & 0,995 & 0,978 & 0,946 & 0,963 \\
\hline \multicolumn{2}{|r|}{ AGFI } & 0,994 & 0,966 & 0,923 & 0,957 \\
\hline \multicolumn{2}{|r|}{ NFI } & 0,995 & 0,968 & 0,921 & 0,958 \\
\hline \multicolumn{2}{|r|}{ RFI } & 0,994 & 0,958 & 0,904 & 0,954 \\
\hline \multicolumn{2}{|r|}{ CFI } & 1 & 0,987 & 0,960 & 0,991 \\
\hline \multicolumn{2}{|r|}{ RMSEA } & 0 & 0,053 & 0,092 & 0,049 \\
\hline \multicolumn{2}{|r|}{ SRMR } & 0,051 & 0,091 & 0,111 & 0,099 \\
\hline \multirow{2}{*}{$\mathrm{F}$} & AVE & 0,757 & & & 0,757 \\
\hline & $\omega$ & 0,980 & & & 0,980 \\
\hline \multirow{2}{*}{ F1 } & AVE & & 0,518 & 0,457 & 0,517 \\
\hline & $\omega$ & & 0,879 & 0,879 & 0,879 \\
\hline \multirow{2}{*}{$\mathrm{F} 2$} & AVE & & 0,641 & 0,641 & 0,641 \\
\hline & $\omega$ & & 0,875 & 0,875 & 0,877 \\
\hline \multirow{2}{*}{ F3 } & AVE & & 0,873 & & 0,870 \\
\hline & $\omega$ & & 0,932 & & 0,931 \\
\hline \multirow{4}{*}{$\mathrm{r}^{2}$} & FyF2 & & & & 0,510 \\
\hline & F1yF2 & & 0,321 & 0,324 & 0,303 \\
\hline & F1yF3 & & 0,236 & & 0,237 \\
\hline & $\mathrm{F} 2 \mathrm{yF} 3$ & & 0,097 & & 0,097 \\
\hline
\end{tabular}

Notas: Modelos: 1F_16i $=$ modelo de un factor con 16 indicadores para EMAF, 3F_13i $=$ modelo de tres factores correlacionados para EMEC con 13 ítems, 2F_13i = modelo de dos factores correlacionados para EMEC con 13 ítems, 4F_29i = modelo de cuatro factores correlacionados para el cuestionario con 29 ítems ( $\mathrm{F}=$ motivos a favor de la $\mathrm{RM}, \mathrm{F} 1=$ miedos, preocupaciones y costos económicos, F2 = aceptación y satisfacción con el estado actual y F3 = falta de información). Índices de ajuste: $\chi^{2}=$ estadístico chi-cuadrado, gl $=$ grados de libertad del modelo, $\chi^{2} / \mathrm{gl}=$ chi-cuadrada relativa, $\mathrm{GFI}=$ índice de bondad de ajuste, $\mathrm{AGFI}=$ índice de bondad de ajuste corregido, NFI = índice normado de ajuste, RFI = índice relativo de ajuste, CFI = índice comparativo de ajuste, RMSEA = error de aproximación cuadrático medio y $\mathrm{SRMR}=$ residuo estandarizado cuadrático medio. Validez convergente de los factores: AVE = varianza media extraída y $\omega=$ omega de McDonald. Validez discriminante: $r^{2}=$ varianza compartida entre los factores. 


\section{EMEC}

El número de factores fue dos por el análisis paralelo y coordenadas óptimas, fue tres por la media de las correlaciones parciales al cuadrado y el número de autovalores mayores que 1. Por la media de las correlaciones parciales a la cuarta potencia fue cuatro.

Tras extraer dos factores y rotar la matriz de cargas factoriales, se configuró un primer factor con nueve indicadores (ítems 17-19, 23, 25-29). El factor presentó consistencia interna buena ( $\alpha$ ordinal $=0,88$ ), una varianza media extraída de 0,46 y una fiabilidad compuesta de 0,88 . Su contenido hace referencia al miedo a la intervención y sus consecuencias, preocupaciones por problemas de salud para operarse y su costo económico y falta de información. El segundo factor quedó conformado por cuatro indicadores (ítems $20-22$ y 24), su consistencia fue buena $(\alpha$ ordinal $=0,88$ ), presentó validez convergente $(\mathrm{AVE}=0,65$ y $\omega=0,88)$, Su contenido hace referencia a la aceptación y satisfacción con el estado actual tras la mastectomía. La varianza compartida entre ambos factores fue del $20,9 \%$. Esta fue menor que la varianza media extraída de cada factor y menor que dos tercios, por lo que los factores presentaron validez discriminante.

Al extraer tres factores, los dos ítems sobre falta de información sobre la RM se separaron del primer factor para formar un tercer factor y el segundo factor permaneció sin cambios y con cargas negativas. El primer factor de miedo a la intervención y sus consecuencias y preocupaciones por problemas de salud para operarse y su costo económico con sus siete indicadores (ítems 17-19, 23 y 25-27) presentó consistencia interna buena $(\alpha$ ordinal $=0,88)$ y validez convergente $(\mathrm{AVE}=0,52$ y $\omega=0,88)$. El segundo factor de aceptación y satisfacción con el estado actual tras la mastectomía mostró consistencia interna buena $(\alpha$ ordinal $=0,88)$ y validez convergente (AVE $=$ $0,65$ y $\omega=0,88)$. El tercer factor de falta de información quedó integrado por dos indicadores (ítems 28 y 29), su consistencia interna fue excelente $(\alpha$ ordinal $=0,93)$ y presentó validez convergente $(\mathrm{AVE}=0,84 \mathrm{y} \omega=0,91)$. La varianza compartida entre los factores varió de 2,1 al 20,8\%, por lo que éstos presentaron validez discriminante.

Se especificaron modelos de dos (Figura 2) y tres factores correlacionados para EMEC (Figura 3). En los dos modelos, la solución fue admisible y todos los parámetros fueron significativos. Los factores del modelo trifactorial presentaron validez convergente (AVE $>0,50$ y $\omega>0,70)$ y validez discriminante $\left(\mathrm{r}^{2}<\right.$ AVE de cada factor $y<2 / 3$ ). En el modelo bifactorial, hubo validez discriminante y el segundo factor mostró validez convergente. El primer factor presentó una varianza media extraída menor que 0,50 , pero su fiabilidad compuesta fue mayor que 0,80 . El ajuste fue mejor en el modelo de tres factores correlacionados. Seis de los ocho índices mostraron buen ajuste y RMSEA y SRMR tuvieron valores de ajuste aceptables. En el modelo de dos factores correlacionados, cuatro índices fueron buenos $\left(\chi^{2} / \mathrm{gl}, \mathrm{GFI}\right.$, AGFI y CFI), tres aceptables (NFI, RFI y RMSEA) y uno malo (SRMR).

Al extraer cuatro factores, conforme al criterio de Velicer revisado (correlaciones parciales a la cuarta potencia), se configuraron los factores de aceptación y satisfacción con el estado actual (ítems 20-22 y 24) y falta de información (ítems 28 y 29). Del factor de miedos, preocupaciones y costos se separaron los ítems 17 y 18 . El 17 sobre miedo a complicaciones tras la reconstrucción con una carga de 0,95 y el ítem 18 sobre costo económico con una carga de 0,49 conformaron el nuevo factor, en el cual ítem 25 sobre miedo al dolor después de la reconstrucción tuvo su segunda carga más alta con un valor de 0,49 . Su contenido versa sobre miedos hacia el futuro por complicaciones. El contenido del factor de miedos y preocupaciones reducido 
a cinco indicadores (ítems 19, 23, 25-27) versa sobre miedos y preocupaciones presentes y futuras. La correlación entre estos dos últimos factores fue alta $(r=0,57$, $\mathrm{p}<0,001)$ en el AFE, En el AFC subió a 0,77, lo provocó un problema de validez discriminante $\left(r^{2}=0,60>\right.$ AVE del nuevo factor $\left.=0,58\right)$. Esta solución se descartó, ya que la separación del factor de miedos, preocupaciones y costos en dos factores resulta forzada y dos factores tienen sólo dos indicadores.

Figura 2. Modelo de dos factores de motivos en contra de la RM estimado por SLS

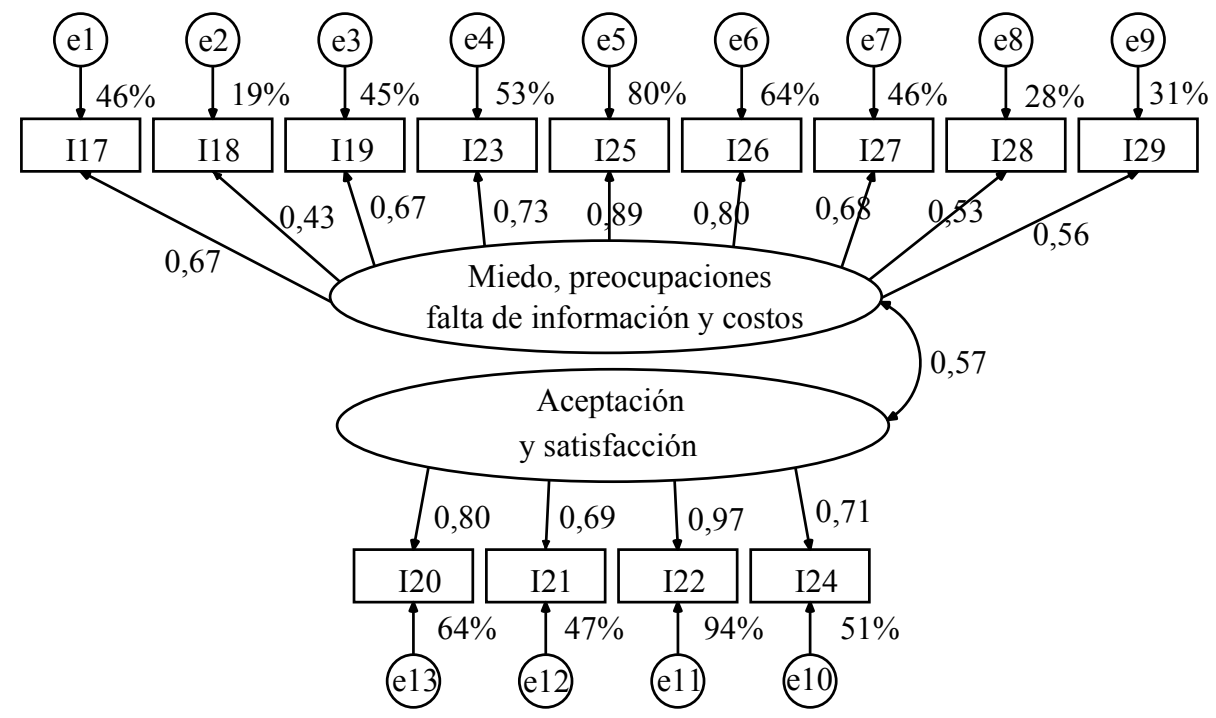

Figura 3. Modelo de tres factores de motivos en contra de la RM estimado por SLS

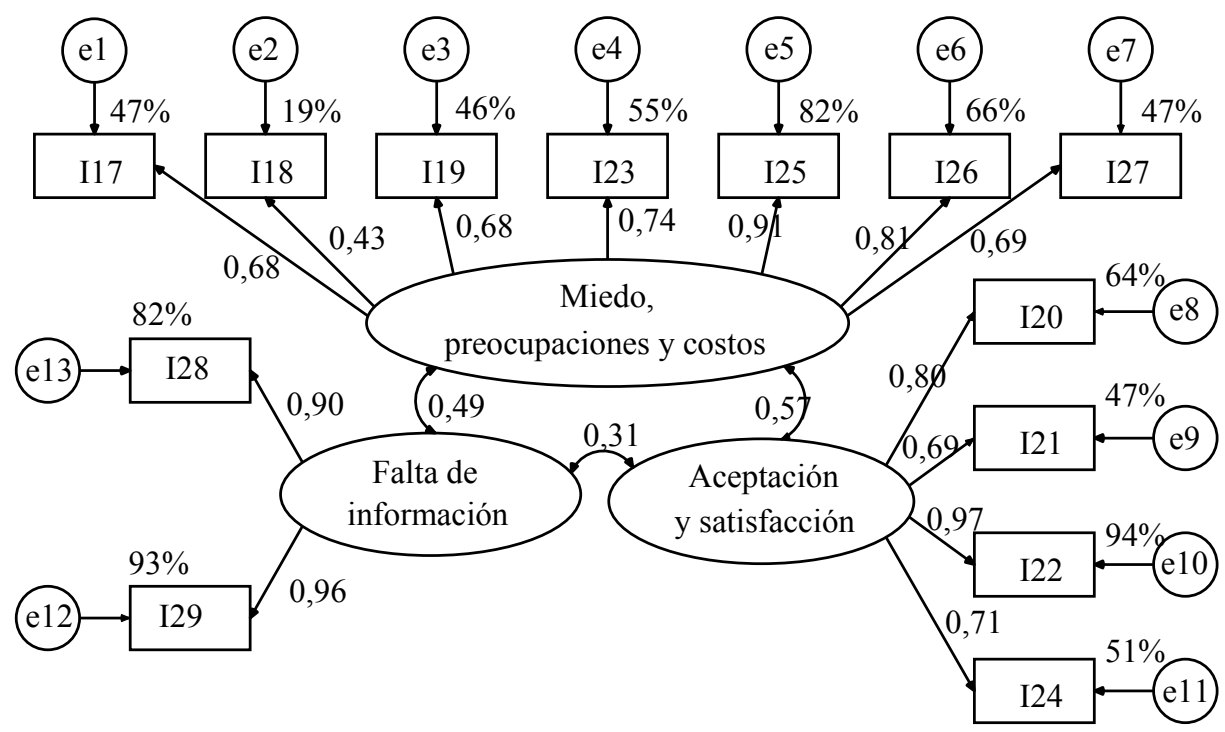




\section{CMRM}

La consistencia interna del CMRM fue excelente $\left(\alpha_{\text {estratificada }}=0,94\right)$. La correlación entre EMAF y EMEC fue significativa y negativa, pero su fuerza de asociación fue pequeña $r=-0,23$, IC 95\% $(-0,42 ;-0,05), t[110]=-2,49, p=0,014$.

Se contrastó un modelo de cuatro factores correlacionados con los 16 ítems de EMAF y 13 de EMEC como modelo para el cuestionario. Las correlaciones del factor de motivos a favor de la RM y los factores de falta de información $(\mathrm{r}=0, \mathrm{p}=$ 1) y miedos, preocupaciones y costos $(r=-0,10, p=0,299)$ no fueron significativas, Tras eliminar estas dos correlaciones, todos los parámetros fueron significativos (Figura 4), Los cuatro factores mostraron validez convergente (AVE $>0,50$ y $\omega>$ $0,80)$ y discriminante $\left(\mathrm{r}^{2}<\right.$ AVE de cada factor y $\left.<0,70\right)$ y el ajuste del modelo fue bueno por siete índices y aceptable por SRMR (Tabla 2).

Figura 4. Modelo de cuatro factores correlacionados para el cuestionario de motivos a favor y en contra de la RM estimado por SLS

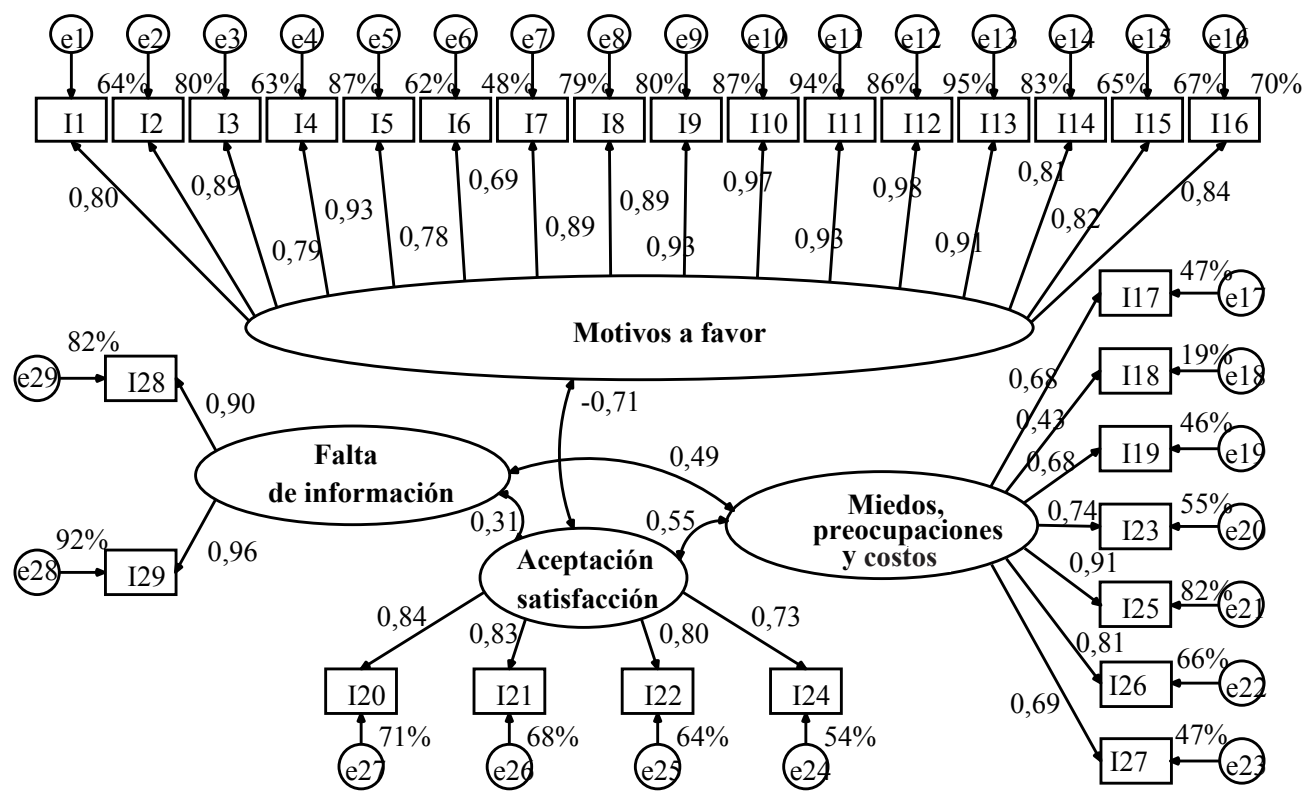

Distribuciones del CMRM, sus dos escalas y los tres factores de la EMEC

Por su mejor ajuste, mayor validez convergente del primer factor y pertinencia del tercer factor, se optó por el modelo de tres factores para la EMEC. Las puntuaciones se interpretaron en sentido absoluto, al dividir los rangos continuos en siete intervalos en correspondencia a los siete valores discretos de respuesta al ítem y de este modo poder usar sus etiquetas de respuesta (Tabla 3 ). 
Tabla 3. Interpretación absoluta de las puntuaciones en el cuestionario, escalas y factores

\begin{tabular}{ccccc}
\hline$[1 ; 7]^{\mathrm{a}}$ & {$[0 ; 1]^{\mathrm{b}}$} & {$[-7 ; 7]^{\mathrm{c}}$} & $\begin{array}{c}\text { Escala ordinal } \\
\text { de respuesta } \\
\text { de los ítems }\end{array}$ & $\begin{array}{c}\text { Etiquetas } \\
\text { de respuesta } \\
\text { de los ítems }\end{array}$ \\
\hline$[1 ; 1,86)$ & {$[0 ; 14)$} & {$[-7 ;-5)$} & 1 & Totalmente en desacuerdo \\
{$[1,86 ; 2,71)$} & {$[0,14 ; 0,29)$} & {$[-5 ;-3)$} & 2 & Bastante en desacuerdo \\
{$[2,71 ; 3,57)$} & {$[0,29 ; 0,43)$} & {$[-3 ;-1)$} & 3 & Algo en desacuerdo \\
{$[3,57 ; 4,43)$} & {$[0,43 ; 0,57)$} & {$[-1 ; 1)$} & 4 & Ni de acuerdo ni en desacuerdo \\
{$[4,43 ; 5,29)$} & {$[0,57 ; 0,71)$} & {$[1 ; 3)$} & 5 & Algo de acuerdo \\
{$[5,29 ; 6,14)$} & {$[0,71 ; 0,86)$} & {$[3 ; 5)$} & 6 & Bastante de acuerdo \\
{$[6,14 ; 7]$} & {$[0,86,1]$} & {$[5 ; 7)$} & 7 & Totalmente de acuerdo \\
\hline
\end{tabular}

Notas: a rango continuo de 1 a 7 para EMAF, EMEC y sus tres factores sin puntuaciones transformadas, ${ }^{\mathrm{b}}$ rango continuo de 0 a 1 para los dos primeros factores del EMEC con la transformación arcoseno de la proporción de las puntuaciones, ${ }^{\mathrm{c}}$ rango continuo de -7 a 7 para la puntuación total en el cuestionario como diferencia entre EMAF y EMEC.

\section{$E M A F$}

La distribución de las puntuaciones en EMAF fue simétrica, pero mostró platicurtosis, por lo que no se ajustó a una distribución normal. Su media aritmética, $\mathrm{M}=4,07$, IC $95 \%(3,69 ; 4,46)$, quedó en el cuarto intervalo de valores $[3,57 ; 4,43)$ que corresponde a una respuesta neutra ("ni de acuerdo ni en desacuerdo"). Su mediana, Mdn = 4,72; quedó en el quinto intervalo $[4,43 ; 5,29)$ que corresponde a una respuesta "algo de acuerdo" (Tabla 4). Se aplicaron varias transformaciones matemáticas a las puntuaciones de la EMEC y la mejor aproximación a la normalidad se consiguió al transformar las puntuaciones en una porción, $\mathrm{p}=(\mathrm{EMAF}-1) / 6$, y aplicar una transformación trigonométrica: EMAFt $=(2 / \pi) * \operatorname{arcsen}\left(p^{1 / 2}\right)$. Con esta transformación, se preservó la simetría, $\mathrm{Sk}=-0,34$, IC 95\% $(-0,80 ; 0,12)$, se atenuó la leptocurtosis, $\mathrm{K}_{3}=-0,93$, IC $95 \%(-1,84 ;-0,03)$, y se mantuvo la hipótesis nula de normalidad con una $\mathrm{p}>0,01$ por la prueba de D'Agostino-Pearson $\left(\mathrm{K}^{2}=6,34\right.$, $\mathrm{p}=0,042)$, pero no por la de Kolmogorov-Smirnov-Lilliefors $\left(\left|\mathrm{D}_{\max }\right|=0,14, \mathrm{p}<\right.$ 0,001), por lo que finalmente no se retomó.

Tabla 4. Estadísticos descriptivos y ajuste a la normalidad

\begin{tabular}{ccccccccc}
\hline Estadísticos & CMRM & EMAF & EMEC & F1 & F1T & F2 & F2T & F3 \\
\hline Min & -6 & 1 & 1 & 1 & 0 & 1 & 0 & 1 \\
Max & 5,85 & 7 & 7 & 7 & 1 & 7 & 1 & 7 \\
& $-0,211$ & 4,072 & 4,283 & 4,282 & 0,536 & 4,039 & 0,500 & 4,773 \\
$M$ & $(-0,74 ;$ & $(3,69 ;$ & $(4,01 ;$ & $(3,96 ;$ & $(0,49 ;$ & $(3,68 ;$ & $(0,45 ;$ & $(4,35 ;$ \\
$($ IC 95\%) & $0,32)$ & $4,46)$ & $4,56)$ & $4,61)$ & $0,58)$ & $4,40)$ & $0,55)$ & $5,19)$
\end{tabular}




\begin{tabular}{|c|c|c|c|c|c|c|c|c|c|}
\hline \multicolumn{2}{|c|}{ Estadísticos } & CMRM & EMAF & EMEC & $\mathrm{F} 1$ & $\mathrm{~F} 1 \mathrm{~T}$ & $\mathrm{~F} 2$ & $\mathrm{~F} 2 \mathrm{~T}$ & F3 \\
\hline \multicolumn{2}{|c|}{$\mathrm{DE}$} & 2,782 & 2,048 & 1,466 & 1,726 & 0,228 & 1,923 & 0,281 & 2,229 \\
\hline \multirow{3}{*}{\multicolumn{2}{|c|}{$\begin{array}{c}\text { Sk } \\
\text { (IC 95\%) }\end{array}$}} & $-0,268$ & $-0,299$ & $-0,288$ & $-0,243$ & $-0,144$ & $-0,049$ & $-0,136$ & $-0,620$ \\
\hline & & $(-0,72$ & $(-0,76$ & $(-0,74$ & $(-0,69$ & $(-0,60$ & $(-0,50$ & $(-0,59$ & $(-1,07$; \\
\hline & & $0,18)$ & $0,16)$ & $0,17)$ & $0,21)$ & $0,31)$ & $0,40)$ & $0,32)$ & $-0,17)$ \\
\hline \multirow{3}{*}{\multicolumn{2}{|c|}{$\begin{array}{c}\mathrm{K}_{3} \\
(\mathrm{IC} 95 \%)\end{array}$}} & $-0,557$ & $-1,383$ & $-0,531$ & $-1,056$ &,- 290 & $-1,135$ &,- 500 & $-1,065$ \\
\hline & & $(-1,45$ & $(-2,29$ & $(-1,44$ & $(-1,95$; & $(-1,19$ & $(-2,03$ & $(-1,40$ & $(-1,96$; \\
\hline & & $0,34)$ & $-0,48)$ & $0,38)$ & $-0,16)$ & $0,61)$ & $-0,24)$ & $0,40)$ & $-0,17)$ \\
\hline \multicolumn{2}{|c|}{$\mathrm{P} 10$} & $-4,362$ & 1 & 2,169 & 1,857 & 0,247 & 1 & 0 & 1 \\
\hline \multicolumn{2}{|c|}{ P20 } & $-2,727$ & 1,450 & 2,877 & 2,286 & 0,306 & 2,25 & 0,302 & 2 \\
\hline \multicolumn{2}{|c|}{$\mathrm{P} 25$} & $-2,159$ & 1,688 & 3,212 & 2,679 & 0,355 & 2,50 & 0,333 & 3 \\
\hline \multicolumn{2}{|c|}{ P30 } & $-1,591$ & 2,706 & 3,400 & 3,286 & 0,424 & 3 & 0,392 & 4 \\
\hline \multicolumn{2}{|c|}{ P40 } & $-0,498$ & 3,888 & 4,108 & 4 & 0,500 & 3,25 & 0,420 & 4,7 \\
\hline \multicolumn{2}{|c|}{ P50 } & 0,166 & 4,719 & 4,385 & 4,571 & 0,561 & 4 & 0,500 & 5,25 \\
\hline \multicolumn{2}{|c|}{$\mathrm{P} 60$} & 0,440 & 5 & 4,815 & 4,857 & 0,592 & 4 & 0,570 & 6 \\
\hline \multicolumn{2}{|c|}{ P70 } & 1,371 & 5,603 & 5,154 & 5,386 & 0,653 & 5,420 & 0,658 & 7 \\
\hline \multicolumn{2}{|c|}{ P75 } & 1,901 & 5,875 & 5,385 & 5,714 & 0,694 & 5,563 & 0,675 & 7 \\
\hline \multicolumn{2}{|c|}{ P80 } & 2,437 & 6,063 & 5,523 & 6 & 0,732 & 6,25 & 0,770 & 7 \\
\hline \multicolumn{2}{|c|}{ P90 } & 3,274 & 6,550 & 6,069 & 6,571 & 0,828 & 6,75 & 0,869 & 7 \\
\hline \multirow[t]{2}{*}{ KSL } & $|D|$ & 0,093 & 0,144 & 0,077 & 0,095 & 0,072 & 0,095 & 0,090 & 0,164 \\
\hline & $\mathrm{p}$ & 0,021 & $<0,001$ & 0,121 & 0,016 & 0,200 & 0,015 & 0,029 & $<0,001$ \\
\hline \multirow[t]{2}{*}{ DP } & $\mathrm{K}^{2}$ & 2,843 & 10,848 & 2,918 & 6,456 & 0,795 & 6,214 & 1,547 & 12,697 \\
\hline & $\mathrm{p}$ & 0,241 & 0,004 & 0,232 & 0,040 & 0,672 & 0,045 & 0,461 & 0,002 \\
\hline
\end{tabular}

Notas: CMRM = cuestionario de motivos a favor y contra la RM = EMAF - EMEC, EMAF = Escala de motivos a favor de la reconstrucción, EMEC = Escala de motivos en contra de la reconstrucción. Factores de EMEC desde el modelo de tres factores: F1 = Miedo a la intervención y sus consecuencias y preocupaciones por problemas de salud para operarse y su costo económico, F2 = Aceptación y satisfacción con el estado actual tras la mastectomía y F3 = Falta de información. Transformación matemática: F1T $=\operatorname{arcseno}\left((2 / \pi)^{*}[(\mathrm{~F} 1-1) / 6]^{1 / 2}\right)$ y $\mathrm{F} 2 \mathrm{~T}=\operatorname{arcseno}\left((2 / \pi)^{*}[(\mathrm{~F} 2-1) / 6]^{1 / 2}\right)$.

\section{$E M E C$}

La distribución de las puntuaciones en EMEC fue simétrica y mesocúrtica, y se ajustó a una distribución normal por las dos pruebas de contraste. Su media aritmética y mediana $(\mathrm{M}=4,28$ y $\mathrm{Mdn}=4,39)$ quedaron en el cuarto intervalo de valores $[3,57$, 4,43) que corresponde a una respuesta neutra (Tablas 3 y 4).

Las distribuciones de las puntuaciones en el primer y segundo factor mostraron simetría, pero ligera platicurtosis, ajustándose a la normalidad por ambas pruebas con una $\mathrm{p}>0,01$. Con la transformación del arcoseno de la proporción de las 
puntuaciones se logró simetría, mesocurtosis y ajuste normalidad por ambas pruebas con una $\mathrm{p}>0,10$. Con esta transformación el rango de las puntuaciones es de 0 a 1 . Para su interpretación en términos absolutos se dividió en siete intervalos (Tabla 3). La media aritmética y mediana de las puntuaciones transformadas del primer factor de miedos, preocupaciones y costos económicos $(\mathrm{M}=0,54$ y $\mathrm{Mdn}=0,56)$ quedaron en el cuarto intervalo de valores $[0,43 ; 0,57)$ que corresponde a una respuesta neutra. A su vez, la media aritmética y mediana del segundo factor de aceptación y satisfacción con el estado actual $(\mathrm{M}=0,50 \mathrm{y} \mathrm{Mdn}=0,50)$ también correspondieron a una respuesta neutra (Tabla 4$)$.

La distribución de las puntuaciones en el tercer factor de falta de información mostró asimetría negativa y platicurtosis, por lo que se rechazó la hipótesis nula de normalidad por ambas pruebas con una $\mathrm{p}<0,01$. Con una transformación de BoxCox potencial $(\mathrm{k}=2)$ y con la transformación trigonométrica, se logró simetría, pero aumentó la platicurtosis, por no se logró normalidad. Consecuentemente, no se retomó ninguna transformación. La media aritmética y mediana $(\mathrm{M}=4,77, \mathrm{y} \mathrm{Mdn}=$ $5,25)$ quedaron en el quinto intervalo de valores que corresponde a una respuesta de "algo de acuerdo" (Tablas 3 y 4).

\section{CMRM}

La distribución de las puntuaciones en el cuestionario se aproximó a una distribución normal. El perfil fue acampado, simétrico y mesocúrtico y la hipótesis nula de normalidad se mantuvo por la prueba de D'Agostino-Pearson con una $\mathrm{p}$ $>0,10$ y por la de Kolmogorov-Smirnov con la correlación para la significación de Lilliefors con una $\mathrm{p}>0,02$. La media aritmética y mediana $(\mathrm{M}=-0,21$ y $\mathrm{Mdn}$ $=0,17)$ tomaron valores muy próximos y reflejaron una disposición motivacional neutra (Tablas 3 y 4). El 34,5\% de las mujeres mostró una disposición motivacional de rechazo $(<1)$, 33,7\% neutra $(\geq-1$ y $<1)$ y $31,18 \%$ de aceptación $(\geq 1)$, siendo los tres porcentajes estadísticamente equivalentes $\left(\chi^{2}[2, \mathrm{~N}=110]=0,13, \mathrm{p}=0,938\right)$.

\subsection{Relación con variables clínicas, imagen corporal, frecuencia de relaciones sexuales y variables sociodemográficas}

Las puntuaciones en EMAF y el tercer factor de EMEC se utilizaron en el rango continuo de 1 a 7 sin asumir normalidad, ya que ninguna transformación matemática logró normalidad. Las puntuaciones en el cuestionario (EMAF - EMEC) y EMEC se utilizaron en el rango continuo de 1 a 7, asumiendo normalidad. Las puntuaciones en el primer y segundo factor de la EMEC se manejaron con la transformación arcoseno asumiendo normalidad.

Las relaciones con las variables cualitativas dicotómicas deseo de conservar la mama, deseo de RM y tipo de mastectomía (unilateral o bilateral) se calcularon a través de la correlación poliserial, salvo con la EMAF y el tercer factor de EMEC que fue por la correlación biserial por rangos.

Las relaciones con las variables ordinales de búsqueda de información sobre la RM, etapa del cáncer, escolaridad, ingreso económico familiar y percepción del estado económico actual se calcularon por la correlación poliserial, salvo con la EMAF y el tercer factor de EMEC que fue por la correlación por rangos ordenados de Spearman. 
La distribución de las puntuaciones en el cuestionario de imagen corporal (CIC) fue simétrica $\mathrm{Sk}=0,36$, IC $95 \%(-0,09 ; 0,82)$ y mesocúrticas $\mathrm{K}_{3}=-0,56$, IC $95 \%$ $(-1,46 ; 0,33)$ y mostró una buena aproximación a la normalidad $\left(\mathrm{K}^{2}=4,01, \mathrm{p}=\right.$ $0,135 ; \operatorname{Max}|\mathrm{D}|=0,09, \mathrm{p}=0,021)$. A su vez, la distribución de la edad fue simétrica, Sk $=0,41$, IC $95 \%(-0,05 ; 0,86)$ y mesocúrtica, $\mathrm{K}_{3}=-0,40$, IC $95 \%(-1,29 ;-0,50)$ y se ajustó a una curva normal (prueba de Kolmogorov-Smirnov con la corrección de Lilliefors: $\operatorname{Max}|\mathrm{D}|=0,08, p=0,057$; prueba de D'Agostino-Pearson: $\mathrm{K}^{2}=3,87$, $\mathrm{p}$ $=0,144)$. Las correlaciones con el CIC y la edad se calcularon por el coeficiente de correlación producto momento de Pearson. En el caso de la EMAF y el tercer factor de EMEC se usó remuestreo repetitivo para estimar la significación e intervalo de las correlaciones.

La relación con el estado civil se estimó a través del coeficiente eta. La significación de esta relación se contrastó por análisis de varianza, salvo con la EMAF y el tercer factor de EMEC que se usó la prueba no paramétrica de Kruskal-Wallis.

El deseo de reconstrucción tuvo una correlación significativa, positiva y con una fuerza de asociación muy grande con el cuestionario y grande con EMAF. Con la EMEC y sus factores, las dos correlaciones significativas fueron negativas; la fuerza de asociación del deseo de reconstrucción fue grande con el factor de aceptación y satisfacción con el estado actual y media con la puntuación total del EMEC. El deseo de conservar la mama tuvo correlaciones significativas y con una fuerza de asociación pequeña. Con el cuestionario y EMAF fueron positivas y con el factor de aceptación y satisfacción del estado actual de la EMEC fue negativa (Tabla 5).

Haberse informado sobre RM tuvo correlaciones significativas y con fuerza de asociación pequeña. Las correlaciones fueron positivas con el cuestionario y EMAF, y fueron negativas con EMEC y su tercer factor (Tabla 5).

Una mayor problemática relacionada con la imagen corporal (CIC) correlacionó positivamente con el cuestionario, EMAF y el tercer factor de la EMEC y negativamente con el segundo factor de la EMEC. La fuerza de asociación fue media, salvo con el tercer factor de la EMEC que fue baja (Tabla 5).

$\mathrm{Al}$ comparar la frecuencia de relaciones sexuales antes del diagnóstico $(M d n=1$ "al menos una vez a la semana") con la frecuencia actual (Mdn = 2 "al menos una vez al mes"), la diferencia fue significativa (prueba de Mann-Whitney: $Z_{U}=-4,98$, $\mathrm{p}<0,001)$ con un tamaño del efecto medio $(\mathrm{r}$ de Rosethal $=0,34)$. La frecuencia de las relaciones sexuales antes del diagnóstico tuvo una correlación significativa, directa y con una fuerza de asociación pequeña con el cuestionario y EMAF, pero fue independiente de EMEC y sus tres factores (Tabla 5).

El cuestionario, EMEC y su primer factor presentaron correlaciones significativas con la percepción del estado económico. La correlación fue negativa con el cuestionario y positiva con EMEC y su primer factor. La fuerza de la asociación fue media con el cuestionario y pequeña en los otros dos casos.

El cuestionario, ambas escalas y el factor de aceptación y satisfacción con el estado actual tras la mastectomía presentaron correlaciones significativas con la edad. Las correlaciones fueron negativas y con una fuerza de asociación media con el cuestionario y EMAF y positiva con una fuerza de asociación media con el segundo factor y pequeña con EMEC (Tabla 5).

El cuestionario, ambas escalas y los tres factores de EMEC fueron independientes de la etapa del cáncer, tipo de mastectomía (unilateral o bilateral), frecuencia de relaciones sexuales actuales, estado civil, escolaridad e ingreso económico familiar. 
Tabla 5. Correlaciones con las variables sociodemográficas y clínicas

\begin{tabular}{|c|c|c|c|c|c|c|}
\hline Variables & CMRM & EMAF & EMEC & F1T & F2T & F3 \\
\hline $\begin{array}{c}\text { Deseo } \\
\text { RM }\end{array}$ & $\begin{array}{l}\mathrm{r}_{\mathrm{pS}}=0,72^{* * *} \\
(0,60 ; 0,84)\end{array}$ & $\begin{array}{l}\mathrm{r}_{\mathrm{BR}}=0,60^{* * *} \\
(0,42 ; 0,77)\end{array}$ & $\begin{array}{c}\mathrm{r}_{\mathrm{PS}}=-0,31^{* *} \\
(-0,53 ;-0,10)\end{array}$ & $\begin{array}{c}\mathrm{r}_{\mathrm{PS}}=-0,09^{\text {ns }} \\
(-0,32 ; 0,15)\end{array}$ & $\begin{array}{c}\mathrm{r}_{\mathrm{PS}}=-0,60^{* * *} \\
(-0,76 ;-0,45)\end{array}$ & $\begin{array}{c}\mathrm{r}_{\mathrm{BR}}<-0,01^{\mathrm{ns}} \\
(-0,22 ; 0,22)\end{array}$ \\
\hline $\begin{array}{c}\text { Deseo } \\
\text { CM }\end{array}$ & $\begin{array}{c}r_{P S}=0,27^{*} \\
(0,05 ; 0,49)\end{array}$ & $\begin{array}{c}r_{B R}=0,25^{* *} \\
(0,04 ; 0,46)\end{array}$ & $\begin{array}{c}\mathrm{r}_{\mathrm{PS}}=-0,05^{\mathrm{ns}} \\
(-0,29 ; 0,20)\end{array}$ & $\begin{array}{c}\mathrm{r}_{\mathrm{PS}}=0,08^{\mathrm{ns}} \\
(-0,16 ; 0,32)\end{array}$ & $\begin{array}{c}\mathrm{r}_{\mathrm{PS}}=-0,21^{*} \\
(-0,43 ;-0,02)\end{array}$ & $\begin{array}{c}\mathrm{r}_{\mathrm{BR}}<0,04^{\mathrm{ns}} \\
(-0,21 ; 0,22)\end{array}$ \\
\hline $\begin{array}{l}\text { Info } \\
\text { RM }\end{array}$ & $\begin{array}{c}r_{\mathrm{s}}=0,26^{* *} \\
(0,07 ; 0,45)\end{array}$ & $\begin{array}{l}r_{s}=0,19^{*} \\
(0 ; 0,40)\end{array}$ & $\begin{array}{c}r_{P S}=-0,22^{*} \\
(-0,42 ;-0,02)\end{array}$ & $\begin{array}{c}\mathrm{r}_{\mathrm{pS}}=-0,12^{\text {ns }} \\
(-0,33 ; 0,09)\end{array}$ & $\begin{array}{c}\mathrm{r}_{\mathrm{ps}}=-0,14^{\mathrm{ns}} \\
(-0,34 ; 0,07)\end{array}$ & $\begin{array}{c}r_{\mathrm{s}}=-0,26^{* *} \\
(-0,47 ;-0,04)\end{array}$ \\
\hline $\begin{array}{l}\text { Etapa } \\
\text { cáncer }\end{array}$ & $\begin{array}{c}r_{P s}=0,03^{\text {ns }} \\
(-0,16 ; 0,23)\end{array}$ & $\begin{array}{c}r_{\mathrm{s}}=-0,02^{\mathrm{ns}} \\
(-0,24 ; 0,19)\end{array}$ & $\begin{array}{c}\mathrm{r}_{\mathrm{PS}}=-0,05^{\mathrm{ns}} \\
(-0,25 ; 0,14)\end{array}$ & $\begin{array}{c}\mathrm{r}_{\mathrm{pS}}=-0,08^{\mathrm{ns}} \\
(-0,28 ; 0,11)\end{array}$ & $\begin{array}{c}r_{p s}=0,06^{\text {ns }} \\
(-0,13 ; 0,25)\end{array}$ & $\begin{array}{c}r_{\mathrm{s}}=-0,05^{\mathrm{ns}} \\
(-0,27 ; 0,17)\end{array}$ \\
\hline Mastect. & $\begin{array}{c}\mathrm{r}_{\mathrm{PS}}=0,05^{\mathrm{ns}} \\
(-0,30 ; 0,39)\end{array}$ & $\begin{array}{c}\mathrm{r}_{\mathrm{s}}=0,06^{\mathrm{ns}} \\
(-0,13 ; 0,25)\end{array}$ & $\begin{array}{c}\mathrm{r}_{\mathrm{PS}}=0,06^{\mathrm{ns}} \\
(-0,28 ; 0,41)\end{array}$ & $\begin{array}{c}\mathrm{r}_{\mathrm{PS}}=0,08^{\mathrm{ns}} \\
(-0,26 ; 0,43)\end{array}$ & $\begin{array}{c}\mathrm{r}_{\mathrm{PS}}=-0,11^{\mathrm{ns}} \\
(-0,45 ; 0,23)\end{array}$ & $\begin{array}{c}\mathrm{r}_{\mathrm{s}}=0,13^{\mathrm{ns}} \\
(-0,06 ; 0,32)\end{array}$ \\
\hline CIC & $\begin{array}{c}r=0,34^{* * *} \\
(0,16 ; 0,52)\end{array}$ & $\begin{array}{c}\mathrm{r}=0,41^{* * *} \\
(0,24 ; 0,57)^{\mathrm{b}}\end{array}$ & $\begin{array}{c}\mathrm{r}=-0,06^{\mathrm{ns}} \\
(-0,25 ; 0,13)\end{array}$ & $\begin{array}{c}\mathrm{r}=0,05^{\mathrm{ns}} \\
(-0,14 ; 0,24)\end{array}$ & $\begin{array}{c}r=-0,32^{* * *} \\
(-0,50 ;-0,14)\end{array}$ & $\begin{array}{c}r=0,18^{*} \\
(0,01 ; 0,35)^{b}\end{array}$ \\
\hline $\begin{array}{l}\text { FRS } \\
\text { antes }\end{array}$ & $\begin{array}{c}r_{P S}=0,24^{*} \\
(0,05 ; 0,43)\end{array}$ & $\begin{array}{c}\mathrm{r}_{\mathrm{s}}=0,25^{* *} \\
(0,07 ; 0,44)\end{array}$ & $\begin{array}{c}\mathrm{r}_{\mathrm{PS}}=-0,10^{\mathrm{ns}} \\
(-0,30 ; 0,10)\end{array}$ & $\begin{array}{c}\mathrm{r}_{\mathrm{PS}}=-0,03^{\mathrm{ns}} \\
(-0,24 ; 0,17)\end{array}$ & $\begin{array}{c}r_{\mathrm{PS}}=-0,09^{\mathrm{ns}} \\
(-0,29 ; 0,11)\end{array}$ & $\begin{array}{c}r_{s}=-0,09^{\text {ns }} \\
(-0,29 ; 0,11)\end{array}$ \\
\hline $\begin{array}{l}\text { FRS } \\
\text { actual }\end{array}$ & $\begin{array}{c}\mathrm{r}_{\mathrm{ps}}=-0,01^{\mathrm{ns}} \\
(-0,21 ; 0,20)\end{array}$ & $\begin{array}{c}\mathrm{r}_{\mathrm{s}}=0,02^{\mathrm{ns}} \\
(-0,19 ; 0,23)\end{array}$ & $\begin{array}{c}\mathrm{r}_{\mathrm{ps}}=0,05^{\mathrm{ns}} \\
(-0,16 ; 0,25)\end{array}$ & $\begin{array}{c}r_{p s}=0,08^{\text {ns }} \\
(-0,13 ; 0,28)\end{array}$ & $\begin{array}{c}\mathrm{r}_{\mathrm{PS}}=0,01^{\mathrm{ns}} \\
(-0,20 ; 0,22)\end{array}$ & $\begin{array}{c}r_{s}=0,08^{n s} \\
(-0,13 ; 0,28)\end{array}$ \\
\hline Edad & $\begin{array}{c}r=-0,37^{* * *} \\
(-0,55 ;-0,19)\end{array}$ & $\begin{array}{c}r=-0,32^{* *} \\
(-0,50 ;-0,14)^{b}\end{array}$ & $\begin{array}{c}r=0,26^{* *} \\
(0,07 ; 0,44)\end{array}$ & $\begin{array}{c}\mathrm{r}=0,17^{\mathrm{ns}} \\
(-0,02 ; 0,36)\end{array}$ & $\begin{array}{c}r=0,33^{* *} \\
(0,15 ; 0,51)\end{array}$ & $\begin{array}{c}\mathrm{r}=0,01^{\mathrm{ns}} \\
(-0,18 ; 0,20)^{\mathrm{b}}\end{array}$ \\
\hline Escol. & $\begin{array}{c}\mathrm{r}_{\mathrm{PS}}=0,05^{\mathrm{ns}} \\
(-0,15 ; 0,24)\end{array}$ & $\begin{array}{c}\mathrm{r}_{\mathrm{s}}=0,02^{\mathrm{ns}} \\
(-0,20 ; 0,24)\end{array}$ & $\begin{array}{c}\mathrm{r}_{\mathrm{PS}}=-0,05^{\mathrm{ns}} \\
(-0,24 ; 0,14)\end{array}$ & $\begin{array}{c}\mathrm{r}_{\mathrm{PS}}=-0,07^{\mathrm{ns}} \\
(-0,26 ; 0,13)\end{array}$ & $\begin{array}{c}\mathrm{r}_{\mathrm{PS}}=-0,04^{\mathrm{ns}} \\
(-0,23 ; 0,15)\end{array}$ & $\begin{array}{c}\mathrm{r}_{\mathrm{s}}=0,05^{\text {ns }} \\
(-0,17 ; 0,27\end{array}$ \\
\hline $\begin{array}{l}\text { Ingreso } \\
\text { económ. }\end{array}$ & $\begin{array}{c}\mathrm{r}_{\mathrm{PS}}=0,08^{\mathrm{ns}} \\
(-0,12 ; 0,28)\end{array}$ & $\begin{array}{c}\mathrm{r}_{\mathrm{s}}=0,04^{\mathrm{ns}} \\
(-0,17 ; 0,26)\end{array}$ & $\begin{array}{c}\mathrm{r}_{\mathrm{PS}}=0,08^{\mathrm{ns}} \\
(-0,28 ; 0,11)\end{array}$ & $\begin{array}{c}\mathrm{r}_{\mathrm{PS}}=-0,04^{\mathrm{ns}} \\
(-0,24 ; 0,15)\end{array}$ & $\begin{array}{c}\mathrm{r}_{\mathrm{PS}}=-0,05^{\mathrm{ns}} \\
(-0,25 ; 0,15)\end{array}$ & $\begin{array}{c}r_{\mathrm{s}}=-0,05^{\mathrm{ns}} \\
(-0,27 ; 0,17)\end{array}$ \\
\hline PEC & $\begin{array}{c}r_{P S}=0,20^{*} \\
(0,01 ; 0,39)\end{array}$ & $\begin{array}{c}\mathrm{r}_{\mathrm{s}}=0,05^{\mathrm{ns}} \\
(-0,17 ; 0,27)\end{array}$ & $\begin{array}{c}r_{\mathrm{PS}}=-0,27^{* *} \\
(-0,47 ;-0,07)\end{array}$ & $\begin{array}{c}r_{\mathrm{PS}}=-0,30^{* *} \\
(-0,49 ;-0,11)\end{array}$ & $\begin{array}{c}r_{\mathrm{PS}}=-0,09^{\text {ns }} \\
(-0,30 ; 0,13)\end{array}$ & $\begin{array}{c}\mathrm{r}_{\mathrm{PS}}=-0,17^{\mathrm{ns}} \\
(-0,39 ; 0,05)\end{array}$ \\
\hline $\begin{array}{c}\text { Estado } \\
\text { civil }\end{array}$ & $\begin{array}{c}\eta=0,20 \\
F=1,13^{\text {ns }}\end{array}$ & $\begin{array}{c}\eta=0,17 \\
\mathrm{H}=6,93^{\text {ns }}\end{array}$ & $\begin{array}{c}\eta=0,12 \\
F=0,39^{\text {ns }}\end{array}$ & $\begin{array}{c}\eta=0,09 \\
F=0,20^{\text {ns }}\end{array}$ & $\begin{array}{c}\eta=0,19 \\
F=0,99^{\text {ns }}\end{array}$ & $\begin{array}{c}\eta=0,11 \\
H=2,65^{\text {ns }}\end{array}$ \\
\hline
\end{tabular}

Notas: $N=110 . \mathrm{CMRM}=$ Cuestionario de motivos a favor y contra la RM =EMAF $-\mathrm{EMEC}$, $\mathrm{EMAF}=$ Escala de motivos a favor de la RM = Suma(I1 a I16)/16. EMEC = Escala de motivos en contra la RM = Suma(I17 a I29)/13, F1T = Puntuaciones transformadas del factor de miedo a la intervención y sus consecuencias, preocupaciones por problemas de salud para operarse $=\operatorname{arcsen}((2 / \pi) *([\mathrm{~F} 1-1] / 7))$, donde $\mathrm{F} 1=(\mathrm{I} 17+\mathrm{I} 18+\mathrm{I} 19+\mathrm{I} 23+\mathrm{I} 25+\mathrm{I} 26+\mathrm{I} 27) / 7$, F2T $=$ Puntuaciones transformadas del factor de aceptación y satisfacción con el estado actual tras la mastectomía $=\operatorname{arcoseno}((2 / \pi) *([\mathrm{~F} 2-1] / 7))$, donde F2 $=(\mathrm{I} 20+\mathrm{I} 21+\mathrm{I} 22+\mathrm{I} 24) / 4$. F3 $=$ Falta de información $=\mathrm{F} 3=(\mathrm{I} 28+\mathrm{I} 29) / 2$. Variables: Deseo $\mathrm{CM}=$ deseo de conservar la mama $(0$ = No y $1=$ Sí), Deseo_RM = deseo de reconstrucción mamaria $(0=$ No y $1=$ Sí $)$, Info_RM $=$ ¿Se ha informado sobre la reconstrucción mamaria?, Mastect. $=$ mastectomía $(0$ $=$ unilateral y $1=$ bilateral $), \mathrm{CIC}=$ cuestionario de imagen corporal, FRS_antes $=$ frecuencia de relaciones sexuales antes del diagnóstico del cáncer, FRS_actual = frecuencia actual de relaciones sexuales y PEC $=$ percepción del estado económico. Coeficientes de asociación con un intervalo de confianza al 95\% (IC 95\%): $r$ = correlación producto-momento de Pearson $\left({ }^{b}=\right.$ estimación por intervalo hecha por el método de percentiles con la simulación de 2.000 muestras aleatorias), $\mathrm{r}_{\mathrm{S}}=$ correlación por rangos de Spearman, $\mathrm{r}_{\mathrm{PS}}=$ correlación poliserial, $\mathrm{r}_{\mathrm{BR}}$ $=$ correlación biserial por rangos de Glass, $\eta=$ coeficiente eta $(F=$ estadístico de contraste por análisis de varianza para grupos independientes y $\mathrm{H}=$ estadístico de contraste por la prueba de Kruskal-Wallis). Significación: ${ }^{n s}=$ no significativa, ${ }^{*} \mathrm{p}<0,05,{ }^{* *} \mathrm{p}<0,01$. 


\section{Discusión}

Se formuló como primer objetivo describir la distribución y comprobar la discriminabilidad y fiabilidad de los ítems de cada escala. Se optó por analizar cada escala por separado, ya que sus ítems fueron generados a partir de pruebas de asociación independientes ${ }^{(19)}$. Se consideró el cuestionario como una composición de ambas escalas y su puntuación como un balance entre los motivos a favor y en contra de la RM. Los 16 ítems de motivos a favor de RM y 12 de los 13 ítems de motivos en contra fueron discriminativos y fiables y ninguno mostró efecto suelo techo o suelo, por lo que todos fueron retenidos para determinar la estructura factorial. El ítem 18 sobre el costo de la intervención como motivo en contra de la RM fue discriminativo, tampoco mostró efecto suelo o techo, pero no cumplió uno de los dos indicadores de fiabilidad. Se optó por retenerlo al ser un tema mencionado con frecuencia en la fase cualitativa del estudio y ser considerado relevante por los jueces ${ }^{(19)}$.

Cabe señalar que todos los ítems tuvieron una distribución en forma de $\mathrm{U}$, con los valores de menor frecuencia en el centro y los de mayor frecuencia en los extremos, lo que sugiere que la mayoría de las mujeres al responder a estas preguntas lo hacen más en términos de aceptación o rechazo que en términos de grados de acuerdo o desacuerdo. No obstante, cabe señalar que la caída en los valores centrales es más abrupta en los ítems a favor de la RM que en los ítems en contra de la RM. Así, parece que motivos a favor elicitan más la polarización que los motivos en contra. Eysenck $^{(32)}$ observó que las escalas que evalúan posicionamiento ideológico poseen este tipo de distribución polarizada en forma de U. Tal vez en la argumentación a favor de la RM entran aspectos ideológicos feministas contra la búsqueda de la felicidad a través de la cirugía cosmética y la subordinación de la estética corporal al servicio del deseo masculino ${ }^{(33)}$ o aspectos de orden moral y conservador ${ }^{(34)}$. Aunque la cirugía estética es un negocio creciente, hoy día despierta mucha polémica y una clara resistencia en instituciones públicas ${ }^{(34)}$.

Aunque la distribución de los ítems tiene una forma de $\mathrm{U}$, sus brazos son disparejos. Dentro de una misma escala en unos ítems el brazo izquierdo es más largo y en otros el derecho, a pesar de ser todos los ítems directos. Con base en la moda, en 11 de los 16 ítems de la EMAF dominó la respuesta "totalmente en desacuerdo" y en 9 de los 13 ítems de la EMEC dominó la respuesta "totalmente de acuerdo", lo que parece inclinar la disposición motivacional hacia el rechazo de la RM. No obstante, con base en la mediana, la tendencia central en la respuesta a los ítems de ambas escalas se ubicaría en una respuesta "algo de acuerdo" o "ni de acuerdo ni en desacuerdo", por lo que el balance a favor o en contra sería ambivalente. Aunque la mediana resulta el valor menos frecuente, finalmente por la forma de $\mathrm{U}$ constituye una mejor medida de la tendencia central y coincide con el promedio que el cuestionario y las escalas arrojan. Cabe remarcar que esta variabilidad a favor o contra la RM que finalmente resulta ambigua es concordante con otros estudios en mujeres mastectomizadas ${ }^{(35)}$, pues es una cuestión polémica que genera conflictos personales e institucionales ${ }^{(34,36)}$.

Se enunció como segundo objetivo determinar la estructura factorial de cada escala. Se inició con un AFE, ya que no existían expectativas sobre el número y contenido de los factores debido al origen empírico de los ítems ${ }^{(19)}$. Luego se calculó el ajuste de los modelos por AFC. Al aplicarse éste sobre la misma muestra que el exploratorio no constituye una prueba adicional de validez de constructo, sino un 
dato complementario al $\mathrm{AFE}^{(25)}$. Finalmente, se integraron los dos modelos para cada escala como propuesta para el CMRM en concordancia con carácter compuesto dado al mismo.

El número de factores fue uno desde los distintos criterios empíricos para los 16 ítems a favor de la RM. El factor único mostró validez convergente en el AFE y AFC, su consistencia interna fue excelente y el ajuste a los datos del modelo unidimensional fue bueno. Las mujeres al valorar los distintos motivos a favor de la RM parecen tener una idea muy clara de su posicionamiento a favor o en contra de esta intervención quirúrgica, lo que determina la unidimensionalidad del factor, su consistencia interna excelente y la forma de U con caída abrupta de la distribución de los ítems.

Con los 13 ítems de la EMEC, el número de factores en un principio no está tan claro. Unos criterios indican dos factores y otros tres factores. Los modelos de dos y tres factores comparten el factor de aceptación y satisfacción con el estado actual tras la mastectomía. La diferencia está en que se separan sus dos ítems sobre falta de información del primer factor y configuran un tercer factor. Este tercer factor a pesar del número insuficiente de indicadores mostró consistencia interna excelente, validez convergente y discriminante tanto en el AFE como AFC sin provocar una solución inadmisible o falta de convergencia. Al ser el ajuste de modelo de tres factores mayor y mejorar la validez convergente del primer factor, se considera que la estructura factorial de tres factores correlacionados representar mejor la estructura subyacente a relación entre los ítems que el modelo de dos factores.

Cabría preguntarse si el problema de consistencia interna del ítem 18 dentro de la EMEC se debe a una formulación deficiente del ítem. Su formulación se basó directamente en las expresiones usadas por mujeres de esta misma población (mastectomizadas por cáncer de mama y atendidas a través del Seguro Popular) y fue revisada por expertos, por lo que no se piensa que ese sea el problema. Otra posibilidad es que sea un indicador de un cuarto factor. Sólo el criterio de Velicer corregido (media de las correlaciones parciales a la cuarta potencia) indicó cuatro factores. Al extraer cuatro factores, el ítem 18 no aparece como indicador único del nuevo factor, sino que, con otro ítem y sin tener el mayor peso de medida, forma un factor sobre miedos hacia el futuro por complicaciones, el cual carece de validez discriminante frente al factor de miedos y preocupaciones. Así, la existencia de un cuarto factor sobre costo económico no es sugerida por los datos entre estas mujeres con Seguro Popular. No obstante, en otra población de mujeres de estrato socio-económico alto y medio-alto, sería bueno explorar un cuarto factor de preocupaciones por el costo económico, para lo que se requeriría incluir más ítems sobre este dominio temático. Por ejemplo, "me preocupa el costo económico de la reconstrucción mamaria" "me preocupa el costo económico de lo que siga a la reconstrucción mamaria", "me preocupa desatender otras necesidades por el gasto de la reconstrucción mamaria" "ya he tenido muchos gastos por el cáncer para meterme en otro más".

Se generó una puntuación total para el cuestionario como balance de ambas escalas a través de su diferencia, ya que se trata de un cuestionario formado por dos escalas que comparten sólo el 5,4\% de su varianza. Consecuentemente, la consistencia interna del cuestionario se calculó por un índice para instrumentos integrados por varias escalas, como es el coeficiente alfa estratificado, y ésta resultó excelente, lo que justifica el uso de una puntuación total. A su vez, un modelo de cuatro factores correlacionados como integración de los modelos para la EMAF y la EMEC 
mostró un buen ajuste a los datos y sus factores contaron con validez convergente y discriminante, lo que proporciona validez de constructo al cuestionario.

El tercer objetivo del estudio fue describir la distribución de las puntuaciones en el CMRM, las escalas (EMAF y EMEC) y los tres factores de la EMEC. La distribución de las puntuaciones en la EMAF y los tres factores de la EMEC no se ajustaron a una curva normal propia de los rasgos de personalidad ${ }^{(37)}$. Considerando la forma en $U$ de los ítems y leptocurtosis de sus distribuciones, se convirtieron sus puntuaciones en proporciones y se aplicó la transformación de arcoseno recomendada para proporciones ${ }^{(38)}$. De este modo se logró normalidad, en los dos primeros factores de la EMEC, resultando estos aptos para el uso de pruebas de contraste paramétrico. Con la EMAF y el tercer factor de la EMEC no se logró. Otra transformación recomendada es la transformación logit ${ }^{(39)}$, pero considerando que el valor modal fue 0 , la transformación arcoseno resulta la mejor opción. Además, la transformación logit tampoco lograba la normalidad deseada. Parece que en la argumentación a favor de la RM entran aspectos ideológicos. Estos podrían ser de índole feministas contra la búsqueda de la felicidad a través de la cirugía cosmética y la subordinación de la estética corporal al servicio del deseo masculino ${ }^{(33)}$ o de índole moral y conservador ${ }^{(34)}$.

Las distribuciones de las puntuaciones en el cuestionario y la escala de motivos en contra de la RM sí siguieron un modelo de distribución normal propio de rasgos de personalidad y actitudes adaptativas. Aunque los 13 ítems de motivos contra la RM presentaron una distribución en forma de U, la caída en sus puntuaciones centrales y alzamiento en los extremos fueron más suaves que en los 16 ítems a favor de la RM. Además, tres décimos de estos ítems se polarizaron hacia la aceptación de la reconstrucción (totalmente en desacuerdo) y siete décimos hacia el rechazo (totalmente de acuerdo), dando lugar su suma a una forma acampanada propia de una distribución normal. Parece que el posicionamiento ideológico se atenúa al valorarse motivos contra la RM y permite una mayor matización. Esta atenuación es más visible si el número de ítems es grande (cuestionario y escala), pero no si el número es pequeño (factores). Precisamente, el factor que más se alejó de la normalidad fue el de falta de información, el cual está integrado por sólo dos ítems, y con este factor la transformación arcoseno y logit no funcionaron.

El efecto de alguna ideología (feminista o conservadora/moral) es propuesto como una conjetura, ya que en el diseño del estudio no se contempló su medida. Fue el análisis, la interpretación de los datos y la literatura sobre $\mathrm{RM}^{(33-34,36)}$, lo que sugirió este posible sesgo. A su vez, tampoco se incluyó ningún instrumento de deseabilidad social que podría arrojar más comprensión sobre las particularidades distribucionales de los ítems y las escalas. La deseabilidad social se puede definir como el sesgo a exagerar las cualidades personales y minusvalorar los propios defectos, ya sea de forma deliberada para generar mejor impresión o de forma automática para fortalecer la autoestima $^{(40)}$.

Se planteó como quinto objetivo estudiar la relación del cuestionario, EMAF y EMEC con variables clínicas, conflictos con la imagen corporal tras la mastectomía, frecuencia de relaciones sexuales y variables sociodemográficas para aportar evidencias de validez de constructo concurrente.

Con respecto a las variables clínicas, se cumplió la expectativa de validez criterial de una asociación muy fuerte entre una disposición motivacional favorable hacia la RM y sí desear la RM. A su vez, más motivos a favor de la RM y menor aceptación 
del estado actual se asocian fuertemente con desear la RM. La asociación con la EMEC fue negativa y moderada, ya que sus otros dos factores fueron independientes. También, conforme a lo esperado para la validez de constructo, deseo de conservar la mama se asoció una disposición motivacional favorable a la RM y más motivos a favor de la RM y menor con aceptación del estado actual. La fuerza de la asociación fue baja y la EMEC resultó independiente, al igual que sus otros dos factores. Precisamente, en estudios de revisión, el deseo de conservar la mama posee un tamaño del efecto pequeño sobre realizarse la $\mathrm{RM}^{(41)}$. La búsqueda de información se asoció a una disposición motivacional favorable hacia la reconstrucción, con motivos a favor de la reconstrucción, con motivos contra la reconstrucción y menor queja de falta de información. Congruente con su contenido, la correlación más alta fue con el factor de falta de información. Aunque las asociaciones fueron bajas, este resultado confirma la asociación débil hallada en estudios previos ${ }^{(35)}$.

El presente estudio no apoya la relación de una disposición motivacional más favorable hacia la RM a una etapa más temprana del cáncer. La etapa del cáncer y la disposición motivacional medida a través de los motivos a favor y en contra fueron independientes como en los estudios sobre expectativas sobre la $\mathrm{RM}^{(35)}$. A su vez, el hecho de sufrir una mastectomía bilateral o unilateral no influye en los motivos a favor o en contra de la RM, lo confirma hallazgos $\operatorname{previos}^{(42)}$.

De las variables sociodemográficas, la edad y la percepción del estado económico fueron las únicas variables con correlaciones significativas. La edad mostró correlaciones medias con el cuestionario, EMAF y el segundo factor de la EMEC y correlación pequeña con EMEC al ser sus otros dos factores independientes. Como se ha observado en estudios previos ${ }^{(5,43,44)}$, las mujeres más jóvenes muestran una mayor motivación para realizarse la RM. Al ser la etapa del cáncer independiente de la motivación para realizarse una RM, pero no la edad de la mujer, los presentes datos indican que el deseo de recuperar una imagen al desnudarse más atractiva, está asociada a la juventud con independencia de la etapa del cáncer o una mayor o menor expectativa de vida.

Hay estudios que reportan una asociación positiva con el ingreso familiar ${ }^{(45)}$. El ingreso económico promedio mensual al mes de la familia durante el último año, es decir el aspecto más objetivo del ingreso, fue independiente tanto de los motivos en contra como a favor, pero no así la percepción del estado económico que se asoció a los motivos en contra. Una valoración subjetiva de peor estado económico se considera como motivo contra la RM y también impacta en una peor disposición motivacional hacia la reconstrucción. No obstante, esta percepción es independiente de los motivos a favor de la RM. Cabe señalar que las mujeres de la muestra tenían Seguro Popular. Este seguro cubre la cirugía en sus aspectos reconstructivos, pero no los implantes mamarios, por tanto pueden obtener un descuento en la intervención, aunque ésta no es gratuita, de ahí que una mala situación económica sí constituye una barrera para hacerse la cirugía reconstructiva. El hecho de que el aspecto objetivo sea independiente, pero no el subjetivo probablemente se deba a que el aspecto objetivo no mide la situación económica de la familia entendida como un balance positivo o equilibrado entre ingresos y gastos. Las cuatro opciones de la percepción del estado económico sí reflejan mejor este balance: tengo dificultades para cubrir mis gastos (balance negativo), tengo dinero para cubrir mis gastos (balance equilibrado), tengo poco dinero para gastos extras (balance equilibrado) y tengo dinero suficiente para gastos extras (balance positivo). 
No se halló asociación lineal positiva con la escolaridad en cuanto que una mayor escolaridad puede favorecer la apertura a procedimientos de reconstructivos y estéticos ${ }^{(44)}$. Las categorías ordenadas definidas fueron ocho y su variabilidad fue grande. Además, se usaron coeficientes de correlación apropiados para la escala de medida ordinal de la variable, por tanto no se puede atribuir a aspectos metodológicos. No obstante, cabe señalar que la fuerza de la asociación entre escolaridad y disposición a realizarse la RM es pequeña en otros estudios ${ }^{(46)}$.

Al igual que en estudios previos ${ }^{(14,16,43)}$, el estado civil fue independiente de los motivos a favor o en contra de la RM. El hecho de que la frecuencia de relaciones sexuales actuales sean independientes de los motivos a favor o en contra de la RM, pero no la frecuencia de relaciones sexuales antes del diagnóstico es concordante con la hipótesis de que el deseo de reanudar la vida sexual es un motivo a favor de la RM con independencia del estado civil ${ }^{(2,44)}$. Así, la frecuencia de relaciones sexuales antes del diagnóstico sólo se asocia con motivos a favor y el cuestionario, pero no con motivos en contra.

Una mayor malestar con la imagen corporal tras la mastectomía se asocia a una disposición motivacional más favorable hacia la reconstrucción, más motivos a favor de la reconstrucción y menor aceptación del estado actual tras la mastectomía, lo que confirma reportes previos ${ }^{(20,47)}$ y da validez a las escalas. Asimismo, las mujeres con más conflictos con su imagen post-mastectomía son las que se quejan de más falta de información como refleja la correlación positiva entre el CIC y el tercer factor de la EMEC.

Como limitación del estudio se tiene el uso de un muestreo no probabilístico por lo que las conclusiones deben ser tomadas con la debida cautela dentro de la población de mujeres mexicanas mastectomizadas por cáncer de mama y que son atendidas a través del Seguro Popular. El tamaño de la muestra fue limitado. No obstante, se contó con más de cinco participantes por ítem en el AFE que es un valor mínimo sugerido ${ }^{(48)}$, y éste es especialmente adecuado cuando las cargas factoriales mayores que $0,50^{(25)}$. Wolf, Harrington, Clark y Miller ${ }^{(49)}$ realizaron un estudio de simulación con 10.000 muestras aleatorias. Con tamaño muestral de 110 se alcanza una potencia mayor que 0,99 en el contraste de un modelo de un factor con 8 indicadores con cargas de 0,65 o 0,80 . La potencia alcanzada es de 0,79 en el contraste de un modelo de tres factores con tres indicadores con pesos de medida de 0,80 y correlaciones entre los factores de 0,30 , y la potencia sería de 0,62 con los pesos de medida de 0,65. A medida que aumenta el número de indicadores, la correlación entre los factores y los pesos de medida mejora la potencia del contraste por AFC. Por tanto, la potencia fue muy buena en el contraste del modelo para la EMAF y aceptable para el modelo de la EMEC.

En conclusión, los perfiles de las distribuciones de los ítems de ambas escalas muestran una forma de $\mathrm{U}$, siendo el valor modal más frecuente, el valor más extremo de rechazo; no obstante, la mediana queda en un valor medio "ni de aceptación ni de rechazo". Los 16 ítems de la EMAF poseen discriminabilidad y fiabilidad a través de la consistencia interna; al igual que 12 de los 13 ítems de la EMEC. Aunque se eleva el valor de consistencia interna de la EMEC con la eliminación del ítem 18 sobre el costo económico, éste resulta discriminativo, su índice de homogeneidad es mayor que 0,30 y es importante para la validez de contenido de la escala, por lo que se justifica su retención. La EMAF presenta una estructura unifactorial con buen ajuste a los datos. Su factor único muestra consistencia interna excelente y validez 
convergente. La estructura de la EMEC es de tres factores correlacionados: temores/ preocupaciones / costos económicos, aceptación / satisfacción con el estado actual y falta de información. Por AFC, los tres factores poseen consistencia interna, validez convergente y discriminante y el ajuste a los datos es bueno. La distribución de la EMAF muestra apuntamiento y no se ajusta a una distribución normal. Su promedio refleja una disposición motivacional ambivalente. La distribución de la EMEC se ajusta a la normalidad. Si las puntuaciones en sus dos primeros factores se convierten en una proporción y se aplica una transformación arcoseno también presentan normalidad, pero la distribución platicúrtica del tercer factor de falta de información no se ajusta a una curva normal aún con esta transformación. Los promedios de la EMEC y sus dos primeros factores muestran también una disposición motivacional ambivalente, pero el promedio del factor de falta de información revela una disposición motivacional de ligero rechazo. La fiabilidad compuesta del cuestionario integrado por las dos escalas con correlación débil es excelente. A su vez, cuenta con validez de constructo estructural. Un modelo de cuatro factores correlacionados definido a partir de la integración de los dos previos presenta buen ajuste a los datos con validez convergente y discriminante en sus factores. Al ser calculada la puntuación en el cuestionario como la diferencia entre ambas escalas, su distribución es normal $\mathrm{y}$ el promedio refleja nuevamente una disposición motivacional ambivalente. El cuestionario y ambas escalas tienen validez de constructo concurrente con el deseo de hacerse la RM. La asociación de la EMEC con este criterio externo se debe a su segundo factor de aceptación del estado actual. El cuestionario y la EMAF también muestran validez de constructo convergente con el deseo de conservar la mama, aunque con este criterio sólo el segundo factor de la EMEC muestra correlación. La frecuencia de relaciones sexuales actuales es independiente de ambas escalas. No obstante, la frecuencia de relaciones sexuales antes del diagnóstico se asocia con una disposición motivacional más favorable hacia la reconstrucción y más motivos a favor de la RM, lo que es concordante con la hipótesis del efecto facilitador del deseo de recuperar la sexualidad previa a la enfermedad. Precisamente, mayor conflicto con la imagen corporal tras la mastectomía motiva una disposición motivacional más favorable hacia la reconstrucción, más motivos a su favor, menor aceptación y satisfacción con el estado actual y más queja de falta de información. Con respecto a las variables sociodemográficas, ser más joven se asocia a una disposición motivacional hacia la RM más favorable, más motivos a favor y menos motivos en contra, siendo mayor la aceptación del estado actual a mayor edad. Una percepción más favorable del estado económico actual se asocia a menos motivos en contra y mejor disposición motivacional hacia la RM. Ambas escalas son independientes de la escolaridad, ingresos económicos (pesos mensuales) y estado civil.

Se sugiere aplicar y seguir estudiando este cuestionario con gran potencial. En estudios futuros se recomienda aumentar el número de indicadores del tercer factor de la EMEC. A tal fin, se puede hacer un grupo focal con mujeres mastectomizadas con el tema de la falta de información y formular las siguientes preguntas abiertas: ¿qué información tienen sobre la reconstrucción mamaria?, ¿indagan sobre la misma o prefieren ignorar esta opción?, ¿cómo la buscan la información? y ¿qué obstáculos encuentran para informarse? Del análisis de contenido de las respuestas, se derivarían los nuevos ítems.

Si las opciones de respuesta a los ítems se reducen a dos, no hay garantías de lograr la normalidad distribucional en la EMEC y sus dos primeros factores, por 
tanto se recomienda seguir usando el formato de escala tipo Likert de siete puntos. En todo caso se podrían reducir las categorías ordenadas de respuesta a cinco. La percepción del estado económico resulta un mejor predictor que el ingreso en pesos al medir balance entre ingresos y gastos, por lo que se recomienda su uso. No se sugiere cambiar la redacción ni eliminar el ítem 18 dentro de esta población, ni aumentar el número de ítems sobre aspectos económicos para definir un cuarto factor, pues éste parece un aspecto poco relevante en la disposición motivacional hacia la RM. Más bien lo económico parece entrar dentro del factor de miedos y preocupaciones en contra de la RM y un ítem puede ser suficiente para su inclusión. No obstante, en otra población, sí se podría explorar un cuarto factor sobre costo económico para la EMEC. Finalmente, se sugiere estudiar la relación del cuestionario con aspectos ideológicos (feminismo, conservadurismo de ala derecha) y la deseabilidad social.

\section{Referencias bibliográficas}

1. Mohar A, Reynoso N, Villarreal-Garza C, Bargalló-Rocha JE, Arce-Salinas C, LaraMedina F. Cáncer de mama en el Instituto Nacional de Cancerología. experiencia del seguro popular 2007-2013. Rev Mex Mastol 2015;5:6-11.

2. Adachi K, Ueno T, Fujioka T, Fujitomi Y, Ueo H. Psychosocial factors affecting the therapeutic decisionmaking and postoperative mood states in Japanese breast cancer patients who underwent various types of surgery: Body image and sexuality. Jpn J Clin Oncol 2007;37:412-8. doi: 10.1093/jjco/hym041

3. Eltahir Y, Lisanne W, Dreise M, van Emmichoven I, Jansen L, Werker P, de Bock G. Quality-of-life outcomes between mastectomy alone and breast reconstruction: comparison of patient-reported BREAST-Q and other health-related quality-of-life measures. Plast Reconstr Surg 2013; 132: 201-9. doi: 10.1097/PRS.0b013e31829586a7

4. Menezes MM, Bello MA, Millen E, Lucas FAS, Carvalho FN, Andrade MFC, Pereira AC, Koifman RJ, Bergmann A. Breast reconstruction and risk of lymphedema after mastectomy: A prospective cohort study with 10 years of follow-up. J Plast Reconstr Aesthet Surg 2016;69:1218-26.

5. Dasgupta P, Youl PH, Pyke C, Aitken JF, Baade PD. Geographical disparity in breast reconstruction following mastectomy has reduced over time. ANZ J Surg 2017;87:183-7. doi.org/10.1111/ans.13710

6. Jeevan R, Cromwell DA, Browne JP, Trivella M, Pereira J, Caddy CM, Sheppard C, van der Meulen JHP. Regional variation in use of immediate breast reconstruction for breast cancer in England. Eur J Surg Oncol 2010;36:750-5. doi: 10.1016/j.ejso.2010.06.008

7. Restrepo C, Estrada J, Mesa G, Arango A, D'amato M, Escobar A, et al. Experiencia de la técnica del ganglio centinela en la cirugía del cáncer de mama, Medellín, Colombia, 2009. Rev Colomb Cir 2011;26:25-32.

8. Greenberg CC, Lipsitz SR, Hughes ME, Edge SB, Theriault R, Wilson JL, Carter WB, Blayney DW, Niland J, Weeks JC. Institutional variation in the surgical treatment of breast cancer: a study of the NCCN. Ann Surg 2011;254:339-45. doi: 10.1097/ SLA.0b013e3182263bb0.

9. Kruper L, Holt A, Xu XX, Duan L, Henderson K, Bernstein L, Ellenhorn J. Disparities in reconstruction rates after mastectomy: patterns of care and factors associated with the use of breast reconstruction in Southern California. Ann Surg Oncol 2011;18:2158-65. doi: 10.1245/s10434-011-1580-z 
10. Fallbjörk U, Rasmussen BH, Karlsson S, Salander P. Aspects of body image after mastectomy due to breast cancer - a two-year follow-up study. Eur J Oncol Nurs 2013;17:340-5. doi: 10.1016/j.ejon.2012.09.002

11. Héquet D, Zarca K, Dolbeault S, Couturaud B, Ngô C, Fourchotte V, et al. Reasons of not having breast reconstruction: a historical cohort of 1937 breast cancer patients undergoing mastectomy. Springer Plus 2013;2: 325-34. doi: 10.1186/2193-1801-2-325

12. López PI, Romeo HR, Bracqbien CS, Ramos A. ¿Por qué las mujeres mastectomizadas no recurren a la reconstrucción mamaria en el sistema de seguridad social? Psicol Salud 2015;25:253-60.

13. Reaby LL. Reasons why women who have mastectomy decide to have or not to have breast reconstruction. Plast Reconstr Surg 1998;101:1810-8.

14. Keith DJW, Walker MB, Walker LG, Heys SD, Sarkar TK, Hutcheon AW, Eremin O. Women who wish breast reconstruction: Characteristics, fears, and hopes. Plast Reconstr Surg 2003;111:1051-6. doi: 10.1097/01.PRS.0000046247.56810.40

15. Lardi AM, Myrick ME, Haug M, Schaefer DJ, Bitzer J, Simmen U, et al. The option of delayed reconstructive surgery following mastectomy for invasive breast cancer: Why do so few patients embrace this offer? Eur J Surg Oncol 2013;39:36-43. doi: 10.1016/j. ejso.2012.08.010

16. Meretoja T, Suominen E. Demand for plastic surgical operations after primary breast cancer surgery. Scand J Surg 2005;94:211-5. doi: 10.1177/145749690509400307

17. Zieliński T, Lorenc-Podgórska K, Antoszewski B. Why women who have mastectomy decide not to have breast reconstruction? Polski Przegląd Chirurgiczny 2014;86:451-5. doi: 10.2478/pjs-2014-0081

18. Manne SL, Topham N, Kirstein L, Virtue SM, Brill K, Devine KA, et al. Attitudes and decisional conflict regarding breast reconstruction among breast cancer patients. Cancer Nurs 2016;39:427-36. doi:10.1097/ncc.0000000000000320

19. Miaja M, Moral J, Villarreal C. Fase cualitativa del desarrollo de una Escala de Motivos a favor y en contra de la Reconstrucción Mamaria (EMRM). Psicooncología 2018;15:32744. doi: 10.5209/PSIC.61439

20. Sebastián J, Manos D, Bueno MJ, Mateos N. Imagen corporal y autoestima en mujeres con cáncer de mama participantes en un programa de intervención psicosocial. Clin Salud 2007; 18:137-61.

21. American Psychological Association. Ethical principles of psychologists and code of conduct. With the 2016 amendment to standard 3.04. Washington, DC: American Psychological Association Press. [Acceso 10 de julio de 2019]. Disponible en https:// www.apa.org/ethics/code/

22. Sociedad Mexicana de Psicología. Código ético del psicólogo. 5a ed. Ciudad de México: Trillas, 2010.

23. World Medical Association. World medical association declaration of Helsinki: ethical principles for medical research involving human subjects. JAMA 2013;310:2191-4. doi: 10.1001/jama.2013.281053

24. Ortiz GM, Díaz PA, Llanos OR, Pérez SM, González K. Dificultad y discriminación de los ítems del examen de metodología de la investigación y estadística. Edumecentro. 2015;7:19-35.

25. Lloret S, Ferreres A, Hernández A, Tomás I. El análisis factorial exploratorio de los ítems: una guía práctica, revisada y actualizada. An Psicol 2014;30: 1151-69. doi: 10.6018/ analesps.30.3.199361. 
26. Courtney MGR. Determining the number of factors to retain in EFA: Using the SPSS R-Menu v2.0 to make more judicious estimations. Prac Assess Res Eval 2013;18:1-14. doi: 10.2147/JHL.S35483

27. Byrne BM. Structural Equation Modelling with AMOS: Basic Concepts, Applications, and Programming. 3rd ed. New York: Routledge, 2016.

28. FornellC,LarckerDF. Structural equation models with unobservablevariables andmeasurement error: algebra and statistics. J Marketing Res 1981;18:382-8. doi: 10.2307/3150980

29. Oliden PE, Zumbo BD. Coeficientes de fiabilidad para escalas de respuesta categórica ordenada. Psicothema 2008;20:896-901.

30. Cho E. Making reliability reliable: A systematic approach to reliability coefficients. Organ Res Methods 2016;19:651-82. doi: 10.1177/1094428116656239

31. Tellez A, García CH, Corral V. Effect size, confidence intervals and statistical power in psychological research. Psychol Russ 2015;8:27-46. doi: 10.11621/pir.2015.0303

32. Eysenck, H. J. The psychology of politics. New York: Routldege, 1998. doi: $10.4324 / 9781351303088$

33. Davis K. Revisiting the feminist debate on cosmetic surgery: some reflections on suffering, agency, and embodied difference. In Heyes CJ, Jones M., editors. Cosmetic surgery: A feminist primer. New York: Routledge. p. 35-48

34. Kerekes R, Walgenbach P. Don't be unhappy, you can be perfect! The institutionalization of aesthetic surgery. In: Weik E, Walgenbach P, editors, Institutions Inc. London: Palgrave Macmillan, 2016. p. 63-93. doi: 10.1057/9781137481498_4

35. Flitcroft K, Brennan M, Spillane A. Women's expectations of breast reconstruction following mastectomy for breast cancer: a systematic review. Support Care Cancer 2017;25:2631-61. doi: 10.1007/s00520-017-3712-x

36. Lin L. Something and nothing: on the psychopolitics of breasts and breastlessness. Studies Gender Sexual 2016;17:45-56. doi: 10.1080/15240657.2016.1135682

37. Tracey TJG. A note on socially desirable responding. J Couns Psychol 2016;63:224-32. doi: $10.1037 / \operatorname{cou} 0000135$

38. Negeri ZF, Shaikh M, Beyene J. Bivariate random-effects meta-analysis models for diagnostic test accuracy studies using arcsine-based transformations. Biom J 2018;60:82744. doi: 10.1002/bimj.201700101

39. Chen K, Cheng Y, Berkout O, Lindhiem O. Analyzing proportion scores as outcomes for prevention trials: a statistical primer. Prev Sci 2017;18:312-21. doi: 10.1007/s11121-0160643-6

40. Wetzel E, Böhnke JR, Brown A. Response biases. In: Leong FTL, Iliescu D, editors (Eds.), The ITC international handbook of testing and assessment. Nw York: Oxford University Press, 2016.p. 349-363

41. Nahabedian MY. Breast reconstruction: A review and rationale for patient selection. Plas Reconstr Surg 2009; 124: 55-62. doi: 10.1097/PRS.0b013e31818b8c23

42. Somogyi RB, Webb A, Baghdikian N, Stephenson J, Edward K, Morrison W. Understanding the factors that influence breast reconstruction decision making in Australian women. Breast 2015;24:124-30. doi: 10.1016/j.breast.2014.11.013

43. Alderman AK, McMahon L, Wilkins EG. The national utilization of immediate and early delayed breast reconstruction and the effect of sociodemographic factors. Plast Reconstr Surg 2003; 111:695-705. doi: 10.1097/01.PRS.0000041438.50018.02

44. Fallbjörk U, Karlsson S, Salander P. Rasmussen BH. Differences between women who have and have not undergone breast reconstruction after mastectomy due to breast cancer. Acta Oncol 2010; 49:174-9. doi: 10.3109/02841860903490069 
45. Morrow M, Scott SK, Menck HR, Mustoe TA, Winchester DP. Factors influencing the use of breast reconstruction postmastectomy: a national cancer database study1. J Am Coll Surg 2001;192:1-8. doi: 10.1016/S1072-7515(00)00747-X

46. Daar DA, Abdou SA, Robinson IS, Levine JP, Thanik V. Disparities in postmastectomy breast reconstruction: A systematic review of the literature and modified framework for advancing research toward intervention. Ann Plast Surg 2018; 81:495-502. doi: 10.1097/ SAP.0000000000001503

47. Latifi M, Salimi S, Barahmand N, Fahimnia F, Allahbakhshian-Farsani L. Postmastectomy information needs and information-seeking motives for women with breast cancer. Adv Biomed Res 2018; 7:75. doi:10.4103/abr.abr_187_17

48. MacCallum RC, Widaman KF, Zhang S, Hong S. Sample size in factor analysis. Psychol Methods 1999;4:84-99. doi:10.1037/1082-989X.4.1.84

49. Wolf EJ, Harrington KM, Clark SL, Miller MW. Sample size requirements for structural equation models: an evaluation of power, bias, and solution propriety. Educ Psychol Meas 2013;76:913-34. doi: 10.1177/0013164413495237 